\title{
Stable isotope ecology of Miocene large mammals from Sandelzhausen, southern Germany
}

\author{
Thomas Tütken · Torsten Vennemann
}

Received: 28 February 2008/ Accepted: 17 July 2008/Published online: 10 February 2009

(C) Springer-Verlag 2009

\begin{abstract}
The carbon, oxygen, and strontium isotope composition of enamel from teeth of large Miocene herbivorous mammals from Sandelzhausen (MN5, late Early/ early Middle Miocene) in the North Alpine foreland basin, were analyzed to infer diet and habitat. The mean enamel $\delta^{13} \mathrm{C}$ value of $-11.4 \pm 1.0 \%(n=53)$ for the nine taxa analyzed (including proboscideans, cervids, suids, chalicotheres, equids, rhinocerotids) indicates a pure $\mathrm{C}_{3}$ plant diet for all mammals. ${ }^{87} \mathrm{Sr} /{ }^{86} \mathrm{Sr}$ ratios of $\sim 0.710$ higher than those from teeth of the western Molasse Basin (0.7080.709) seem to indicate preferential feeding of the mammals in the northeastern Molasse Basin. The sympatric herbivores have different mean $\delta^{13} \mathrm{C}$ and $\delta^{18} \mathrm{O}$ values which support diet partitioning and/or use of different habitats within a $C_{3}$ plant ecosystem. Especially the three sympatric rhinoceroses Plesiaceratherium fahlbuschi, Lartetotherium sansaniense, and Prosantorhinus germanicus show clear partitioning of plants and/or habitats. The palaeomerycid Germanomeryx fahlbuschi was a canopy folivore in moderately closed environments whereas Metaschizotherium bavaricum (Chalicotheriidae) and $P$. germanicus (Rhinocerotidae) were browsers in more closed forest environments. The horse Anchitherium aurelianense was probably a more generalized feeder than assumed from its dental morphology. The forest hog
\end{abstract}

\section{T. Tütken $(\bowtie)$}

Steinmann Institut für Geologie, Mineralogie und Paläontologie, Poppelsdorfer Schloss, University of Bonn, 53115 Bonn, Germany

e-mail: tuetken@uni-bonn.de

T. Tütken $\cdot$ T. Vennemann

Institut de Géochimie, Université de Lausanne,

1015 Lausanne, Switzerland

e-mail: Torsten.Vennemann@unil.ch
Hyotherium soemmeringi has the highest $\delta^{13} \mathrm{C}$ and lowest $\delta^{18} \mathrm{O}$ value of all analyzed taxa, possibly related to a frugivorous diet. Most taxa were water-dependent browsers that record meteoric water $\delta^{18} \mathrm{O}$ values of about $-5.6 \pm 0.7 \%$ Vienna Standard Mean Ocean Water (VSMOW). Using a modern-day mean annual air temperature (MAT) $-\delta^{18} \mathrm{O}_{\mathrm{H}_{2} \mathrm{O}}$ relation a MAT of $19.3 \pm 1.5^{\circ} \mathrm{C}$ can be reconstructed for Sandelzhausen. A Gomphotherium subtapiroideum tusk serially sampled for $\delta^{18} \mathrm{O}$ values does not record a clear pattern of seasonality. Thus most taxa were $\mathrm{C}_{3}$ browsers in a forested and humid floodplain environment in the Molasse Basin, which experienced a warm-temperate to subtropical climate and possibly low seasonality.

Keywords Carbon isotopes - Oxygen isotopes ·

Strontium isotopes - Mean annual air temperature .

Enamel $\cdot$ Diet $\cdot$ Drinking water $\cdot$ Molasse Basin

Kurzfassung Die Kohlenstoff-, Sauerstoff- und Strontium-Isotopenzusammensetzung von Zahnschmelzproben miozäner herbivorer Großsäuger aus Sandelzhausen (MN5, Unter-/Mittelmiozän) im nördlichen Alpenvorlandbecken wurde analysiert, um die Ernährungsweise und den Lebensraum zu rekonstruieren. Der mittlere Zahnschmelz $\delta^{13} \mathrm{C}$-Wert von $-11,4 \pm 1,0 \%$ o $(n=53)$ für die 9 analysierten Taxa (u.a. Rüsseltiere, Hirsche, Schweine, Klauentiere, Pferde, Nashörner) zeigt, dass alle Säugetiere eine ausschließlich $\mathrm{C}_{3}$-Pflanzen basierte Ernährungsweise hatten. Die ${ }^{87} \mathrm{Sr} /{ }^{86} \mathrm{Sr}-$ Verhältnisse von $\sim 0,710$, die höher sind als von Zähne aus dem westlichen Molasse Becken (0,707-0,709), deuten auf eine bevorzugte Nahrungsaufnahme im Bereich des nordöstlichen Molasse Beckens hin. Die zeitgleich vorkommenden Herbivoren haben unterschiedliche $\delta^{13} \mathrm{C}$ - und $\delta^{18} \mathrm{O}$-Werte, welche 
auf die Nutzung unterschiedlicher Nahrungsressourcen und/oder Lebensräume innerhalb eines $\mathrm{C}_{3}$-Ökosystems hindeuten. Insbesondere die drei Nashörner Plesiaceratherium fahlbuschi, Lartetotherium sansaniense und Prosantorhinus germanicus zeigen die Nutzung verschiedener Nahrungspflanzen und/oder Habitate. Der Palaeomerycide Germanomeryx fahlbuschi war ein Unterholz-Blattfresser in weniger geschlossenen Habitaten während Metaschizotherium bavaricum (Chalicotheriidae) und Prosantorhinus germanicus (Rhinocerotidae) Laubfresser in eher geschlossenen Wäldern waren. Das Pferd Anchitherium aurelianense war vermutlich ein mehr generalistischer Pflanzenfresser als aufgrund seiner Zahnmorphologie bisher angenommen. Das Waldschwein Hyotherium soemmeringi hat die höchsten $\delta^{13} \mathrm{C}$ und niedrigsten $\delta^{18} \mathrm{O}$-Werte aller analysierten Taxa, was möglicherweise auf eine frugivore Ernährungsweise hindeutet. Die meisten Taxa waren wasserabhängige Blattfresser, die $\delta^{18} \mathrm{O}-$ Werte des meteorischen Wassers von $-5,6 \pm 0,7 \%$ VSMOW aufgezeichnet haben. Unter Nutzung einer modernen mittleren Jahrestemperatur (MAT)$\delta^{18} \mathrm{O}_{\mathrm{H}_{2} \mathrm{O}}$-Relation kann eine MAT von $19,3 \pm 1,5^{\circ} \mathrm{C}$ für Sandelzhausen rekonstruiert werden. Ein seriell für $\delta^{18} \mathrm{O}$-Werte beprobter Gomphotherium subtapiroideum Stoßzahn zeigt kein klares Saisonalitätsmuster. Daher waren die meisten Taxa $\mathrm{C}_{3}$-Blattfresser in einer bewaldeten, feuchten Überflutungsebene im Molasse Becken mit einem warmtemperierten bis subtropischen Klima möglicherweise ohne stark ausgeprägte Saisonalität.

Schlüsselwörter Kohlenstoffisotopie .

Sauerstoffisotopie - Strontiumisotopie ·

Mittlere Jahrestemperatur · Zahnschmelz · Nahrung ·

Trinkwasser - Molasse Becken

\section{Introduction}

Next to palaeontological evidence such as dental features, dental wear, stomach contents, and coprolithes, the geochemical evidence revealed in stable carbon and oxygen isotope analysis of fossil tooth enamel has become an important tool in reconstructing palaeodiet and palaeoecology (see overviews in Kohn and Cerling 2002; Koch 2007). These geochemical systems allow for inferences to be made on diet, feeding behavior, and resource partitioning among taxa as well as drinking behavior and water use efficiency of mammals within modern and ancient ecosystems (e.g., Longinelli 1984; Kohn et al. 1996, 1998, 2005; Cerling et al. 1997a, b, 2003a; Sponheimer and LeeThorp 1999a; MacFadden et al. 1999; Levin et al. 2006). Studies have shown that mammalian skeletal remains (i.e., tooth enamel) record in their isotope composition the vegetation and habitat in which the particular taxa lived (e.g., van der Merwe and Medina 1991; Cerling et al. 2004; Kohn et al. 2005). In contrast to bone, tooth enamel is suited for such geochemical reconstructions as it reliably reflects the original chemical and isotopic composition over millions of years (Schoeninger and DeNiro 1982; LeeThorp and van der Merwe 1987; Wang and Cerling 1994; Kohn et al. 1999). Most carbon isotope studies aiming to reconstruct the palaeodiet and niche partitioning of mammals have focused on environments capable of distinguishing $\mathrm{C}_{3}$ browsers and $\mathrm{C}_{4}$ grazers (e.g., Quade et al. 1992; Cerling et al. 1997b). However, recent studies have also shown enamel carbon isotopes useful in modern and ancient $\mathrm{C}_{3}$-dominated ecosystems to infer niche partitioning (Quade et al. 1995; Cerling et al. 1997a, 2003b, 2004; MacFadden and Higgins 2004; Feranec and MacFadden 2006; Feranec 2007; Nelson 2007; Zanazzi and Kohn 2008). Due to climatic and environmental conditions $\mathrm{C}_{3}$ plant ecosystems prevailed in Europe during Palaeogene and Neogene and no $\mathrm{C}_{4}$ grasslands developed (Blondel et al. 1997; Cerling et al. 1997b). Therefore $C_{4}$ plants were not a significant component of the flora and thus not an important food source for herbivores.

The reconstructions of palaeodiet and palaeoenvironment for the late Early/early Middle Miocene vertebrate fossil locality of Sandelzhausen, Bavaria, southern Germany, have mainly focused on geological, palaeontological, and sedimentological evidence (e.g., Fahlbusch et al. 1972, 1974; Fahlbusch and Liebreich 1996; Schmid 2002; Moser et al. 2009b, this issue) and dental wear (Kaiser and Rössner 2007; Schulz et al. 2007; Schulz and Fahlke 2009, this issue). In this study we attempt to infer resource use and partitioning of taxa at the Sandelzhausen site using stable carbon, oxygen, and strontium isotopes of the enamel of different sympatric large herbivorous mammals such as proboscideans, rhinocerotids, equids, cervids, and suids.

\section{Geological setting}

The Miocene fossil locality of Sandelzhausen, a former gravel pit, is situated $70 \mathrm{~km}$ north of Munich in the North Alpine Foreland Basin (Fig. 1). The fossiliferous sediments are part of the limnofluvial Upper Freshwater Molasse. On the basis of biostratigraphical correlation using micromammals Sandelzhausen is placed in the early part of the European Land Mammal Zone MN5 (Heissig 1997). This corresponds to an age (between 16 and $16.5 \mathrm{Ma}$ ) close to the Early/Middle Miocene boundary (Heissig 1997; AbdulAziz et al. 2008; Moser et al. 2009b, this issue).

The depositional environment was a low-relief, fluvial plain with sedimentary conditions varying between river channels, flood plains, and riparian ponds and lakes. The 


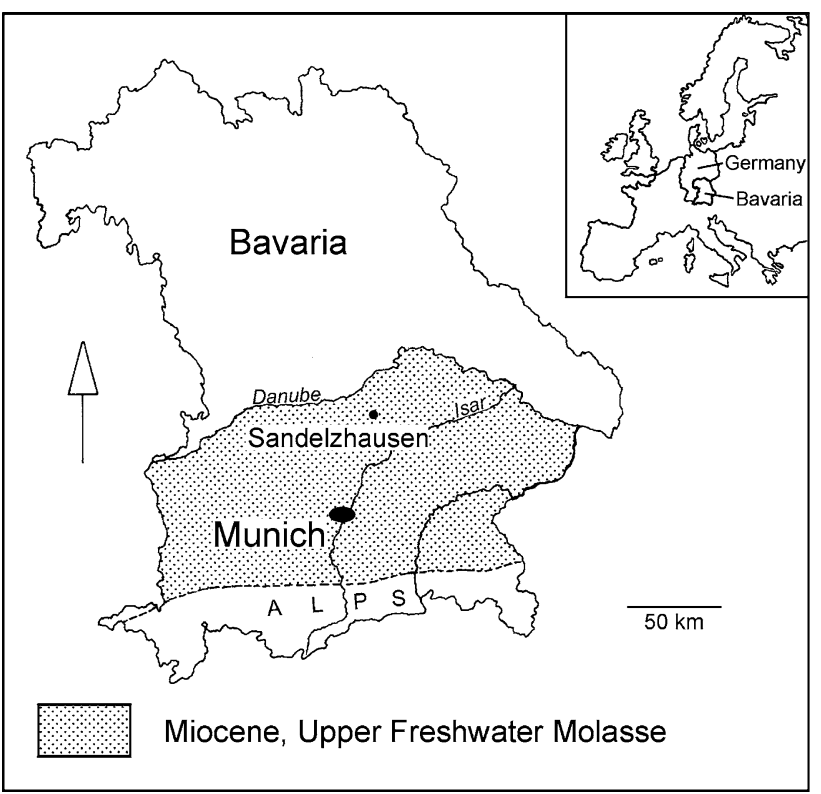

Fig. 1 Fossil locality of Sandelzhausen in the North Alpine Foreland basin. Map from Göhlich (2002)

fossil-bearing limnofluvial sediments consist of a 2-3-mthick succession of gravels to marls with variable but quickly decreasing gravel content toward the top of the section (Fahlbusch et al. 1972, 1974; Schmid 2002; Moser et al. 2009b, this issue). Soil formation processes occurred and the sediment is partly cemented by pedogenic carbonates (Schmid 2002). The sediment and fossil assemblage was probably deposited within a relatively short interval and no time averaging of the faunal remains is recognized. Deposition took place in two freshwater settings under high groundwater level but with different hydrologic regimes: (1) the marly gravel of the lower part of the section occurred in a fluvial floodplain environment with seasonally fluctuating groundwater tables and flood events from a large river in the vicinity, (2) the marly upper part occurred in a lacustrine setting of a permanent, shallow, and stagnant riparian pond with a relatively stable groundwater table (Böhme 2009, this issue; Moser et al. 2009a, this issue).

The lower half of the section includes a humus-enriched black to brown or violet-colored layer of $10-35 \mathrm{~cm}$ thickness (layer C in Fahlbusch et al. 1972; Moser et al. $2009 \mathrm{~b}$, this issue). The mammal teeth analyzed in this study derive from both stratigraphic units, below and above layer $\mathrm{C}$. The skeletal remains were likely carcasses washed in with floods or represent remnants of crocodile prey, indicated by frequent bite marks on the fossil bones (Heissig 2005, personal communication). Therefore, this site is represented by almost only disarticulated skeletal elements such as jaw fragments, teeth or single bones. About 50,000 disarticulated vertebrate remains were discovered and collected along with some plant and abundant invertebrate fossils, including 69 species of gastropods, bivalves, and ostracods (Witt 1998; Fahlbusch 2003; Moser et al. 2009a, this issue). Most of the fauna consists of micro- and macrovertebrates including teleost fish, amphibians, turtles, squamats, crocodilians, birds, and 66 mammal species (Fahlbusch 2003; Moser et al. 2009b, this issue). The mammals include: rhinocerotids, gomphotheriids, suoids, equids, palaeomerycids, cervids, and tragulids. The richness of this terrestrial macro- and microvertebrate fauna (in total more than 200 taxa) makes Sandelzhausen an exceptional site (Fahlbusch 2003; Moser et al. 2009b, this issue). One unique characteristic of the vertebrate fossil assemblage is the high percentage of juvenile individuals present for many taxa.

The invertebrate fauna of gastropods, ostracods, and bivalves are typical for shallow, temperate to subtropical freshwater conditions in the aquatic depositional environment (Moser et al. 2009a, this issue; Witt 1998). Land snail taxa are indicative of humid habitats close to the edge of the water body. Plant remains, however, are scarce or poorly preserved. Only gyrogonites of stoneworts and some fruits of Celtis and leaflets of Podogonium were found (Fahlbusch et al. 1972; Gregor 1982). The vegetation in the vicinity of Sandelzhausen can thus only be reconstructed based on the fossil plant remains from other, nearby Miocene deposits. Such studies support seasonally humid, warm-temperate to subtropical climate conditions, with evergreen to deciduous forests (subtropical oak-laurel forest) and woodlands (Gregor 1982; Schweigert 1992; Jechorek and Kovar-Eder 2004; Böhme et al. 2007).

Based on current knowledge, the environment can be characterized as a humid, periodically flooded riverplain of a braided river system with temporary ponds and lakes, and soil and pedogenic carbonate formation (Schmid 2002) with a more or less closed forest cover. Habitat variability between forest and open gravel-sandbar dominated environments can be expected as implied by the vertebrate faunas (e.g., Böhme 2009, this issue). A mean annual precipitation (MAP) of $564 \pm 252 \mathrm{~mm}$ in the lower part of the section and of $847 \pm 254 \mathrm{~mm}$ in the upper part of the section as well as a mean annual air temperature (MAT) ranging from $18^{\circ} \mathrm{C}$ to $20.8^{\circ} \mathrm{C}$ for the Sandelzhausen setting were estimated from ectothermic-vertebrate-based transfer functions (Böhme et al. 2006; Böhme 2009, this issue).

\section{The use of stable isotopes in palaeoecology}

\section{Carbon isotopes}

The carbon isotope composition $\left(\delta^{13} \mathrm{C}\right)$ of fossil vertebrates is informative for both palaeodietary and palaeohabitat 
reconstructions because of differences in carbon isotope compositions of plants, which are transferred to body tissues of the fauna feeding on them (DeNiro and Epstein 1978). The differences in plant carbon isotope compositions are mostly due to different photosynthetic pathways used for carbon assimilation (O'Leary 1988; Farquhar et al. 1989). Most terrestrial plants assimilate atmospheric $\mathrm{CO}_{2}$ with either the $\mathrm{C}_{3}$ or $\mathrm{C}_{4}$ photosynthetic pathway. $\mathrm{C}_{3}$ plants, which include most trees and shrubs, and grasses favored by cool, wet growing seasons, utilize the enzyme ribulose bisphosphate carboxylase-oxygenase (Rubisco) to fix $\mathrm{CO}_{2}$, forming a three-carbon sugar. $\mathrm{C}_{4}$ plants, which include mostly grasses and sedges growing in warm, dry habitats, use a different enzyme to fix $\mathrm{CO}_{2}$, the phosphoenolpyruvate (PEP) carboxylase, resulting in a four-carbon acid (Farquhar et al. 1989). Both photosynthetic pathways fractionate the light ${ }^{12} \mathrm{C}$ carbon isotopes to a different degree (Farquhar et al. 1989). Therefore, the $C_{3}$ and $C_{4}$ photosynthetic pathway yield different ${ }^{13} \mathrm{C} /{ }^{12} \mathrm{C}$ ratios in plant tissues. Modern $\mathrm{C}_{3}$ plants have a mean $\delta^{13} \mathrm{C}$ value of $-27 \%$ and range between $-22 \%$ and $-36 \%$, while modern $\mathrm{C}_{4}$ plants have a mean $\delta^{13} \mathrm{C}$ value of $-13 \%$ and range between $-10 \%$ and $-15 \%$ (Deines 1980; O'Leary 1988). Crassulacean acid metabolism (CAM) plants, using the CAM photosynthetic pathway, include succulents (Cactaceae) and exhibit a wide range of $\delta^{13} \mathrm{C}$ values that can overlap between those of $\mathrm{C}_{3}$ and $\mathrm{C}_{4}$ plants (Deines 1980; Farquhar et al. 1989). CAM plants represent only a minor fraction of the overall plant biomass though, and are not considered to be an important food resource for herbivores studied from Sandelzhausen.

The distinct $\delta^{13} \mathrm{C}$ values of $\mathrm{C}_{3}$ and $\mathrm{C}_{4}$ plants (mostly $\mathrm{C}_{4}$ grasses) are often used in palaeoecological and palaeodietary studies to distinguish browsers from grazers in ecosystems where both types of plants are present (e.g., Cerling et al. 1997a; MacFadden et al. 1999). The occurrence of a $\mathrm{C}_{4}$ component in the diet of herbivorous mammals is interpreted as the use of $\mathrm{C}_{4}$ grass and thus feeding in open grasslands. However, this approach is only applicable to settings in which $\mathrm{C}_{4}$ grasses occur. A differentiation of browsers and grazers in a pure $\mathrm{C}_{3}$ ecosystem based on enamel $\delta^{13} \mathrm{C}$ values alone is not possible. The first grass fossils occur in rocks about $50 \mathrm{Ma}$ (Palaeocene/ Eocene; Crepet and Feldman 1991), but grasslands did not become globally widespread until the Miocene (Jacobs et al. 1999). The first fossils of $\mathrm{C}_{4}$ grasses with the typical Kranz microanatomy were found in Middle Miocene deposits ( $\sim 10 \mathrm{Ma})$ of North America (Tidwell and Nambudiri 1989). Based on carbon isotope analysis of fossil mammal teeth and ancient soil carbonates, $\mathrm{C}_{4}$ plants do only occur as a major component in global ecosystems since the late Miocene $(\sim 7 \mathrm{Ma})$ when $\mathrm{C}_{4}$ grasslands developed on most continents, excluding Europe (Cerling et al. 1993, 1997b). $\mathrm{C}_{4}$ grasses are thus not expected to form a major food source for the late Early/early Middle Miocene mammals from Sandelzhausen in Central Europe, but $\mathrm{C}_{3}$ grasses may have occurred.

Significant and detectable variability of carbon isotope compositions exists even within pure $\mathrm{C}_{3}$-plant-dominated ecosystems, because of variations in light intensity, temperature, nutrient, and water availability, all of which influence the $\delta^{13} \mathrm{C}$ value in $\mathrm{C}_{3}$ plants (Ehleringer et al. 1986, 1987; Farquhar et al. 1989; Ehleringer and Monson 1993; Heaton 1999). Most $\mathrm{C}_{3}$ plants have $\delta^{13} \mathrm{C}$ values between $-25 \%$ and $-29 \%$ and average about $-27 \%$ (Deines 1980; Farquhar et al. 1989). However, in densely forested ecosystems subcanopy plants growing in closedcanopy rain forests have very low $\delta^{13} \mathrm{C}$ values between $-32 \%$ and $-36 \%$ because of low irradiance and ${ }^{13} \mathrm{C}$-depleted $\mathrm{CO}_{2}$ from biomass degradation near the forest floor (van der Merwe and Medina 1989, 1991; Cerling et al. 2004). In contrast, water-stressed $C_{3}$ plants in open and/or arid environments have more positive $\delta^{13} \mathrm{C}$ values of up to $-22 \%$ and in rare cases even higher because they can close their stomata to decrease water loss and in so doing inhibit $\mathrm{CO}_{2}$ transport and fractionate less against ${ }^{13} \mathrm{C}$ (Farquhar et al. 1989; Cerling et al. 2004). Such differences in plant carbon isotope compositions are transferred to the fauna feeding on these plants. Therefore, such environmentally induced carbon isotope differences in $\mathrm{C}_{3}$ plants can be used to discriminate resource partitioning and habitat use of vertebrates in modern and ancient $\mathrm{C}_{3}$ environments (Quade et al. 1995; Blondel et al. 1997; Drucker et al. 2003; Cerling et al. 2003b, 2004; MacFadden and Higgins 2004; Feranec and MacFadden 2006; Feranec 2007; Nelson 2007; Drucker et al. 2008; Zanazzi and Kohn 2008).

Plant carbon ingested by herbivores is metabolized and incorporated into the mineralized skeletal tissues of animals in the form of carbonate ion $\left(\mathrm{CO}_{3}^{2-}\right)$ that substitutes to 2-4 wt.\% (Driessens and Verbeeck 1990) for the $\mathrm{PO}_{4}^{3-}$ and $\mathrm{OH}$ group in the enamel hydroxy apatite $\left(\mathrm{Ca}_{5}\left(\mathrm{PO}_{4}\right)_{3} \mathrm{OH}\right)$. Skeletal carbonate hydroxyapatite forms in isotopic equilibrium with the blood and its dissolved inorganic carbon and the bioapatite carbonate is enriched in ${ }^{13} \mathrm{C}$ several permille relative to ingested isotope values (Tieszen and Fagre 1993; Passey et al. 2005). An extensive field study of wild East African large herbivorous ungulates (including grazers and browsers as well as foregut and hindgut fermenters) found an average ${ }^{13} \mathrm{C}_{\text {enamel-diet }}$ enrichment factor of $14.1 \pm 0.5 \%$ relative to the plant diet (Cerling and Harris 1999). However, in a recent controlled feeding study Passey et al. (2005) found different ${ }^{13} \mathrm{C}_{\text {enamel-diet }}$ enrichment factors of $13.3 \pm 0.3 \%$ for nonruminant pigs and $+14.6 \pm 0.3 \%$ for ruminant cows raised on an isotopically identical diet. Therefore, digestive physiology (e.g., the 
rate of ${ }^{12} \mathrm{C}$-rich methane production and its loss) can have an important influence on the ${ }^{13} \mathrm{C}_{\text {enamel-diet }}$ enrichment factor (Passey et al. 2005). Enamel $\delta^{13} \mathrm{C}$ values mostly reflect the diet of the animal but may be additionally affected to some degree by its digestive physiology (Passey et al. 2005; Zanazzi and Kohn 2008). Such a physiological influence may become increasingly important when trying to interpret isotope values in an ecosystem where animals are ingesting plants that are isotopically less variable, such as $\mathrm{C}_{3}$-dominated ecosystems.

Carbon isotope values are interpreted based upon certain cutoff values for diets of $\mathrm{C}_{3}$ versus $\mathrm{C}_{4}$ vegetation as well as for closed versus open habitats. The $\delta^{13} \mathrm{C}$ values for enamel apatite of modern pure $\mathrm{C}_{3}$ feeding mammals have a general range from approximately $-8 \%$ to $-22 \%$, with the extremes representing distinctive habitats, low values closed-canopy rainforest and high values open xeric habitats (Cerling et al. 1997b, 2004; Cerling and Harris 1999). In terms of habitat for modern mammals, low enamel $\delta^{13} \mathrm{C}$ values $(-22 \%$ to $-14 \%$ ) are indicative for feeding in mesic closed-canopy forests (Cerling et al. 2004), intermediate values $(-13 \%$ to $-8 \%$ ) for feeding in woodlands and open woodlands, and high values (around $-8 \%$ ) for feeding in xeric $\mathrm{C}_{3}$ grasslands (Cerling and Harris 1999; Bocherens 2003). In contrast, pure $\mathrm{C}_{4}$ feeders have higher $\delta^{13} \mathrm{C}$ enamel values of $-1 \%$ to $+4 \%$. Intermediate values $\left(-8 \%\right.$ to $-1 \%$ ) would characterize mixed $\mathrm{C}_{3}$ and $\mathrm{C}_{4}$ plant feeders (Cerling and Harris 1999).

As modern atmospheric $\mathrm{CO}_{2}\left(\delta^{13} \mathrm{C}_{\mathrm{CO}_{2}}=-8 \%\right.$ is $1.5 \%$ depleted in ${ }^{13} \mathrm{C}$ compared with preindustrial $\mathrm{CO}_{2}$ with a $\delta^{13} \mathrm{C}$ value of $-6.5 \%$, due to the fossil-fuel burning of ${ }^{12} \mathrm{C}$-rich hydrocarbons (Friedli et al. 1986), in this study we assume a $\delta^{13} \mathrm{C}_{\mathrm{CO}_{2}}$ value of $-6.5 \%$ for the Miocene atmosphere. Miocene $\mathrm{C}_{3}$ and $\mathrm{C}_{4}$ plants assimilating such $\mathrm{CO}_{2}$ are then expected to have mean $\delta^{13} \mathrm{C}$ values of $-25.5 \%$ (range $-20.5 \%$ to $-34.5 \%$ ) and $-11.5 \%$ (range $-8.5 \%$ to $-13.5 \%$ ), respectively. Applying the average ${ }^{13} \mathrm{C}_{\text {apatite-diet }}$ enrichment factor of $+14.1 \%$ or large ungulates (Cerling and Harris 1999), enamel $\delta^{13} \mathrm{C}$ values for large Miocene mammalian herbivores feeding in a pure $\mathrm{C}_{3}$ ecosystem should have a mean value of $-11.4 \%$ and a range from $-6.4 \%$ to $-20.4 \%$.

Taxa that fed in more closed and mesic habitats are expected to have more negative carbon isotope values relative to those feeding in more open and/or arid environments (Cerling et al. 2004; Kohn et al. 2005). The most negative $\delta^{13} \mathrm{C}$ values are expected to occur in close, dense forest environments due to the canopy effect, the recycling of isotopically light carbon in the form of $\mathrm{CO}_{2}$ from the degrading biomass (van der Merwe and Medina 1989, 1991; Cerling et al. 2004). Subcanopy ungulates can have about $6 \%$ lower $\delta^{13} \mathrm{C}$ values than gap-clearing folivores, for example (Cerling et al. 2004). Therefore, in $\mathrm{C}_{3}$-plant- dominated ecosystems the effect of environments (e.g., open versus closed, humid versus arid, lit versus shaded) on the $\delta^{13} \mathrm{C}$ values of food plants and thus herbivore body tissues is important.

Enamel reliably reflects the carbon isotope values that the animal ingested (e.g., Lee-Thorp and van der Merwe 1987; Wang and Cerling 1994; Lee-Thorp and Sponheimer 2005). Therefore, the carbon isotope composition of fossil enamel allows for palaeodietary and palaeoenvironmental reconstructions (see reviews in 2007; Kohn and Cerling 2002).

\section{Oxygen isotopes}

The oxygen isotope composition $\left(\delta^{18} \mathrm{O}_{\mathrm{H}_{2} \mathrm{O}}\right)$ of meteoric water varies within ecosystems due to changes in air temperature and/or amount of precipitation or evaporation (Dansgaard 1964; Rozanski et al. 1993). These oxygen isotope differences in meteoric water, which is used as drinking water by mammals, are recorded in their skeletal tissues, that form in isotope equilibrium with the body water, and can be used to infer climatic conditions such as air temperature and aridity as well as animal drinking behavior (Longinelli 1984; Kohn 1996; Kohn and Cerling 2002; Levin et al. 2006; Tütken et al. 2006). Most of the oxygen in the enamel apatite is bound as phosphate $\left(\mathrm{PO}_{4}^{3-}\right)$ and carbonate $\left(\mathrm{CO}_{3}^{2-}\right)$ ions. The major portion of this is in the phosphate group, as the carbonate rarely makes up more than 2-4 wt.\% (Driessens and Verbeeck 1990). The phosphate $\left(\delta^{18} \mathrm{O}_{\mathrm{PO}_{4}}\right)$ and carbonate $\left(\delta^{18} \mathrm{O}_{\mathrm{CO}_{3}}\right)$ oxygen isotope composition of bone and enamel apatite are positively correlated and have an equilibrium offset of about $8.5 \%$ (Bryant et al. 1996; Iacumin et al. 1996). Therefore, both the $\delta^{18} \mathrm{O}_{\mathrm{PO}_{4}}$ and $\delta^{18} \mathrm{O}_{\mathrm{CO}_{3}}$ values reflect the isotopic composition of ingested water.

Herbivorous mammals derive their water from three sources: surface water, water from food, and metabolic water from food processing during oxidation of carbohydrates (Bryant and Froelich 1995; Kohn 1996). The body water $\delta^{18} \mathrm{O}$ value of obligate drinkers, such as most large mammals, is linearly related to that of the drinking water (Longinelli 1984; Kohn 1996; Kohn et al. 1996). However, in addition to rainwater $\delta^{18} \mathrm{O}$ values, several other physiological, environmental, and behavioral factors can affect enamel $\delta^{18}$ O values (e.g., Kohn 1996; Kohn et al. 1996). An important factor is the water dependency of the animal (Levin et al. 2006). Mammals that drink frequently have enamel $\delta^{18} \mathrm{O}$ values that are dependent on rainwater $\delta^{18} \mathrm{O}$ values whereas drought-tolerant animals usually have higher $\delta^{18} \mathrm{O}$ values (Ayliffe and Chivas 1990; Levin et al. 2006) because they obtain proportionally more water from ${ }^{18} \mathrm{O}$-enriched food sources such as leaves, fruits or seeds. Water in plant roots and stems is isotopically similar to 
meteoric water, but leaf water is relatively enriched in $\mathrm{H}_{2}^{18} \mathrm{O}$ due to preferential evapotranspiration of the lighter $\mathrm{H}_{2}{ }^{16} \mathrm{O}$ molecule (Dongmann et al. 1974; Epstein et al. 1977; Sternberg 1989; Yakir 1992, 1997). Thus, diet can have a strong effect on the body water oxygen isotope composition, so that sympatric herbivores may have $\delta^{18} \mathrm{O}$ values that can differ by as much as $8-9 \%$ (Bocherens et al. 1996; Kohn et al. 1996; Sponheimer and Lee-Thorp 1999b). Browsing taxa often have higher relative ${ }^{18} \mathrm{O}$ content compared with grazing taxa of the same age and region, because they ingest a higher proportion of ${ }^{18} \mathrm{O}$ enriched water with their food (Kohn et al. 1996; Sponheimer and Lee-Thorp 1999b).

The higher the water dependency of a terrestrial animal the closer its body water $\delta^{18} \mathrm{O}$ values are to that of meteoric water (Kohn 1996; Clementz and Koch 2001; Levin et al. 2006). Taxa inhabiting preferentially closed-canopy forests or swampy environments will have lower enamel $\delta^{18} \mathrm{O}$ values because of the high humidity of these environments and thus decreased leaf water ${ }^{18} \mathrm{O}$-enrichment due to evapotranspiration (e.g., Cerling et al. 2004). Furthermore, aquatic or semiaquatic animals such as marine mammals or hippopotamuses have lower $\delta^{18} \mathrm{O}$ values than sympatric terrestrial mammals (Bocherens et al. 1996; Clementz and Koch 2001). Therefore, $\delta^{18} \mathrm{O}$ values can allow for inferences on the habitat properties, feeding ecology, drinking behavior, and humidity (Ayliffe and Chivas 1990; Kohn et al. 1998; Sponheimer and Lee-Thorp 1999b; Levin et al. 2006; Zanazzi and Kohn 2008).

In addition mammal teeth mineralize over several months to years. While mineralizing, they record seasonal changes in the isotope composition of ingested food and water (e.g., Koch et al. 1989; Fricke and O’Neil 1996; Kohn et al. 1996). However, the seasonal amplitudes of these changes are dampened due to enamel maturation after initial tooth mineralization (Passey and Cerling 2002). Nevertheless, seasonality is recorded and $\delta^{18} \mathrm{O}$ amplitude changes allow for an evaluation of climatic changes (Fricke et al. 1998; Sharp and Cerling 1998; Nelson 2005, 2007).

\section{Materials and methods}

\section{Material}

In this study the carbon and oxygen isotope compositions of bulk enamel carbonate samples of 53 large mammal teeth from Sandelzhausen (all stored at the Bayerische Staatssammlung für Paläontologie und Geologie in Munich under collection number BSPG 1959 II) were analyzed (Tables 1, 2). The sampled teeth belong to nine different large mammal species including: the cervid Heteroprox eggeri, the palaeomerycid Germanomeryx fahlbuschi, the chalicothere Metaschizotherium bavaricum, the proboscidean Gomphotherium subtapiroideum, the equid Anchitherium aurelianense, the three rhinoceroses, Prosantorhinus germanicus, Plesiaceratherium fahlbuschi, and Lartetotherium sansaniense, and the suid Hyotherium soemmeringi. In addition to samples of the bulk enamel, the enamel growth zones of one ever-growing G. subtapiroideum tusk were sampled. Sixteen serial enamel samples were taken every $2-4 \mathrm{~mm}$, perpendicular to the growth axis of the tusk (Table 3 ). The oxygen isotope composition of enamel phosphate $\left(\delta^{18} \mathrm{O}_{\mathrm{PO}_{4}}\right)$ was measured for four teeth, one Anchitherium, two rhinoceros, and one Gomphotherium molars (Table 4). Finally, for four teeth, including one Anchitherium molar and three Gomphotherium molars, also the strontium isotope compositions $\left({ }^{87} \mathrm{Sr} /{ }^{86} \mathrm{Sr}\right.$ ) were measured (Table 5).

Bulk enamel samples were taken along the complete crown height available, drilling parallel to the growth axis of the tooth using a handheld Proxxon minidrill with diamond-studded drill tips. Therefore bulk sample (30$50 \mathrm{mg}$ ) represents the average isotope composition over the interval of tooth formation, likely encompassing several months to 2 years (Kohn 2004), depending on species, tooth type, and wear pattern. Mostly third and second molar teeth -in some cases also premolars-were sampled to retrieve postweaning dietary and drinking water isotope compositions. With the exception of one sample, first molars (M1) that mineralize wholly or partly before weaning were avoided because they could be potentially biased by isotope effects of maternal milk during consumption (Bryant et al. 1994). However, for several specimens specific molar identification was not possible. Therefore, we cannot exclude the possibility of having sampled some first molars.

\section{Methods}

\section{$C$ and $O$ isotope measurements of the carbonate in the apatite}

Isotopic analysis for all samples was done using $10 \mathrm{mg}$ enamel powder, which was chemically pretreated according to methods given by Koch et al. (1997) to remove organics, and diagenetic carbonate $2 \% \mathrm{NaOCl}$ solution was used for $24 \mathrm{~h}$, followed by a $1 \mathrm{M} \mathrm{Ca}$-acetate acetic acid buffer solution for another $24 \mathrm{~h}$, prior to analysis of the carbon $\left(\delta^{13} \mathrm{C}\right)$ and oxygen $\left(\delta^{18} \mathrm{O}_{\mathrm{CO}_{3}}\right)$ isotopic composition of the carbonate in the apatite. About 2-3 mg pretreated enamel powder was reacted with $100 \% \mathrm{H}_{3} \mathrm{PO}_{4}$ for $90 \mathrm{~min}$ at $70^{\circ} \mathrm{C}$ using a ThermoFinnigan Gasbench II (Spötl and Vennemann 2003). Carbon and oxygen isotope ratios of the generated $\mathrm{CO}_{2}$ were measured in continuous flow mode on a Finnigan Delta Plus XL isotope ratio gas mass 
Table $1 \delta^{13} \mathrm{C}$ and $\delta^{18} \mathrm{O}$ values of enamel apatite

\begin{tabular}{|c|c|c|c|c|c|c|c|c|c|}
\hline Sample & Specimen nr. & Species & $\begin{array}{l}\text { Tooth } \\
\text { position }\end{array}$ & $\begin{array}{l}\delta^{13} \mathrm{C} \\
\text { VPDB } \\
(\%)\end{array}$ & SD & $\begin{array}{l}\delta^{18} \mathrm{O} \\
\text { VPDB } \\
(\% \text { ) }\end{array}$ & SD & $\begin{array}{l}\delta^{18} \mathrm{O} \\
\text { VSMOW } \\
(\%)\end{array}$ & $\begin{array}{l}\mathrm{CaCO}_{3} \\
\text { (wt. } \% \text { ) }\end{array}$ \\
\hline FZ CE SA 1 & BSPG 1959116619 & Heteroprox eggeri & $\mathrm{m} 3$ & -12.0 & 0.04 & -7.7 & 0.12 & 23.0 & 4.0 \\
\hline FZ CE SA 3 & BSPG 1959116621 & Heteroprox eggeri & $\mathrm{m} 3$ & -10.6 & 0.04 & -9.4 & 0.10 & 21.3 & 3.7 \\
\hline FZ CE SA 4 & BSPG 1959114146 & Heteroprox eggeri & $\mathrm{m} 3$ & -10.4 & 0.05 & -6.0 & 0.11 & 24.7 & 3.8 \\
\hline FZ CE SA 7 & BSPG 1959114164 & Heteroprox eggeri & $\mathrm{m} 2$ & -11.1 & 0.07 & -9.2 & 0.10 & 21.5 & 4.2 \\
\hline FZ CE SA 8 & BSPG 1959 II 5196 & Germanomeryx fahlbuschi & M & -11.1 & 0.05 & -7.7 & 0.11 & 23.0 & 4.4 \\
\hline FZ CE SA 9 & BSPG 1959 II 5202 & Germanomeryx fahlbuschi & M dext. & -12.4 & 0.03 & -5.0 & 0.12 & 25.8 & 4.7 \\
\hline FZ CE SA 10 & BSPG 1959 II 5186 & Germanomeryx fahlbuschi & M & -12.6 & 0.09 & -4.5 & 0.11 & 26.3 & 4.6 \\
\hline FZ CH SA 1 & BSPG 1952 II 11620 & Metaschizotherium bavaricum & M & -12.3 & 0.07 & -7.9 & 0.06 & 22.8 & 4.0 \\
\hline FZ CH SA 2 & No nr. & Metaschizotherium bavaricum & $\mathrm{P} 2$ or $\mathrm{P} 3$ & -12.8 & 0.04 & -7.8 & 0.08 & 22.9 & 3.9 \\
\hline FZ CH SA 3 & BSPG 1959 II 11628 & Metaschizotherium bavaricum & $\mathrm{m} 1$ & -13.9 & 0.03 & -7.1 & 0.11 & 23.6 & 4.9 \\
\hline FZ CH SA 4 & BSPG 1959 II 11629 & Metaschizotherium bavaricum & $\mathrm{p} 4$ & -12.6 & 0.05 & -8.3 & 0.09 & 22.3 & 4.9 \\
\hline FZ CH SA 5 & BSPG 1959 II 11630 & Metaschizotherium bavaricum & M & -11.9 & 0.05 & -6.3 & 0.14 & 24.4 & 4.6 \\
\hline FZ MA SA 1 & BSPG 1959 II 11416 & Gomphotherium subtapiroideum & I2 dext. & -11.9 & 0.13 & -9.6 & 0.20 & 21.0 & 4.1 \\
\hline FZ MA SA 2 & BSPG 1959 II 11326 & Gomphotherium subtapiroideum & D4 dext. & -11.5 & 0.08 & -8.8 & 0.14 & 21.8 & 3.5 \\
\hline FZ MA SA 3 & BSPG 1959 II 44 & Gomphotherium subtapiroideum & M & -11.5 & 0.06 & -7.2 & 0.08 & 23.5 & 3.2 \\
\hline FZ MA SA 4 & No nr. & Gomphotherium subtapiroideum & M & -11.2 & 0.05 & -8.2 & 0.09 & 22.4 & 4.0 \\
\hline FZ MA SA 5 & No nr. & Gomphotherium subtapiroideum & M & -10.6 & 0.04 & -8.3 & 0.12 & 22.3 & 4.8 \\
\hline FZ MA SA 6 & No nr. & Gomphotherium subtapiroideum & M & -10.2 & 0.06 & -6.8 & 0.09 & 23.9 & 5.0 \\
\hline FZ MA SA 7 & No $\mathrm{nr}$. & Gomphotherium subtapiroideum & M & -10.8 & 0.05 & -7.5 & 0.12 & 23.2 & 7.5 \\
\hline FZ EQ SA 1 & BSPG 1959 II 577 & Anchitherium aurelianense & $\mathrm{P} 2$ & -11.4 & 0.10 & -6.6 & 0.19 & 24.1 & 4.0 \\
\hline FZ EQ SA 2 & No $\mathrm{nr}$. & Anchitherium aurelianense & $\mathrm{M}$ or $\mathrm{P}$ & -9.5 & 0.04 & -6.5 & 0.10 & 24.3 & 3.6 \\
\hline FZ EQ SA 3 & No nr. & Anchitherium aurelianense & M & -11.2 & 0.05 & -7.7 & 0.12 & 22.9 & 3.6 \\
\hline FZ EQ SA 4 & No nr. & Anchitherium aurelianense & M & -11.3 & 0.05 & -6.0 & 0.13 & 24.7 & 3.6 \\
\hline FZ EQ SA 5 & No $\mathrm{nr}$. & Anchitherium aurelianense & $\mathrm{M}$ or $\mathrm{P}$ & -11.7 & 0.05 & -7.9 & 0.09 & 22.7 & 4.4 \\
\hline FZ EQ SA 6 & No nr. & Anchitherium aurelianense & M & -11.8 & 0.08 & -5.5 & 0.13 & 25.2 & 4.1 \\
\hline FZ EQ SA 7 & No nr. & Anchitherium aurelianense & M & -10.6 & 0.07 & -5.7 & 0.08 & 25.0 & 3.9 \\
\hline FZ EQ SA 8 & BSPG 1959 II 5215 & Anchitherium aurelianense & M & -11.1 & 0.04 & -7.0 & 0.09 & 23.6 & 4.2 \\
\hline FZ RH SA 1a & BSPG 1959116793 & Plesiaceratherium fahlbuschi & $\mathrm{m} 3$ & -11.3 & 0.04 & -6.3 & 0.06 & 24.4 & 4.5 \\
\hline FZ RH SA 1c & BSPG 1959116793 & Plesiaceratherium fahlbuschi & $\mathrm{m} 1$ & -11.6 & 0.06 & -7.5 & 0.07 & 23.2 & 5.4 \\
\hline FZ RH SA 2a & BSPG 1959114416 & Plesiaceratherium fahlbuschi & $\mathrm{p} 4$ & -11.0 & 0.05 & -7.7 & 0.06 & 23.0 & 4.5 \\
\hline FZ RH SA 2b & BSPG 1959114416 & Plesiaceratherium fahlbuschi & p3 & -11.1 & 0.07 & -7.8 & 0.13 & 22.9 & 4.6 \\
\hline FZ RH SA 3 & BSPG 1959115149 & Plesiaceratherium fahlbuschi & $\mathrm{m} 3$ & -11.4 & 0.04 & -6.0 & 0.07 & 24.7 & 5.1 \\
\hline FZ RH SA 4 & BSPG 195911 3530a & Plesiaceratherium fahlbuschi & $\mathrm{m} 3$ & -11.9 & 0.04 & -7.7 & 0.11 & 22.9 & 5.6 \\
\hline FZ RH SA 5 & BSPG 1959117002 & Plesiaceratherium fahlbuschi & $\mathrm{m} 2$ & -12.0 & 0.04 & -7.4 & 0.12 & 23.3 & 5.2 \\
\hline FZ RH SA 6 & BSPG 1959116748 & Plesiaceratherium fahlbuschi & $\mathrm{m} 3$ & -12.5 & 0.03 & -7.3 & 0.12 & 23.4 & 3.6 \\
\hline FZ RH SA 7a & No nr. & Lartetotherium sansaniense & $\mathrm{m} 3$ & -11.5 & 0.04 & -7.8 & 0.13 & 22.9 & 4.3 \\
\hline FZ RH SA 7b & No nr. & Lartetotherium sansaniense & $\mathrm{m} 2$ & -11.6 & 0.06 & -7.7 & 0.09 & 23.0 & 5.0 \\
\hline FZ RH SA 8 & No nr. & Lartetotherium sansaniense & $\mathrm{m} 3$ & -10.7 & 0.05 & -7.1 & 0.08 & 23.6 & 4.6 \\
\hline FZ RH SA 9 & BSPG 1959113817 & Lartetotherium sansaniense & $\mathrm{P} 2$ & -10.2 & 0.02 & -6.5 & 0.11 & 24.2 & 4.7 \\
\hline FZ RH SA 10 & BSPG 1959116742 & Prosantorhinus germanicus & $\mathrm{p} 4$ & -12.9 & 0.05 & -6.0 & 0.07 & 24.7 & 4.1 \\
\hline FZ RH SA 11 & No nr. & Prosantorhinus germanicus & M3 & -11.8 & 0.04 & -8.1 & 0.10 & 22.6 & 4.4 \\
\hline FZ RH SA 12 & BSPG 1959 II 2595 & Prosantorhinus germanicus & M3 & -13.6 & 0.03 & -7.1 & 0.09 & 23.6 & 4.7 \\
\hline FZ RH SA 13 & BSPG 1959 II 2676 & Prosantorhinus germanicus & M2 & -11.7 & 0.05 & -7.1 & 0.08 & 23.6 & 4.5 \\
\hline FZ RH SA 14 & No nr. & Prosantorhinus germanicus & P3 & -12.5 & 0.04 & -7.6 & 0.12 & 23.1 & 4.2 \\
\hline FZ RH SA 15 & No nr. & Prosantorhinus germanicus & M3 & -12.1 & 0.05 & -7.4 & 0.10 & 23.3 & 3.9 \\
\hline FZ SU SA 1 & BSPG 195911311 & Hyotherium soemmeringi & M2 & -10.8 & 0.05 & -9.8 & 0.14 & 20.8 & 3.9 \\
\hline FZ SU SA 2 & BSPG 195911238 & Hyotherium soemmeringi & $\mathrm{m} 3$ & -9.6 & 0.04 & -8.2 & 0.06 & 22.5 & 4.6 \\
\hline
\end{tabular}


Table 1 continued

\begin{tabular}{|c|c|c|c|c|c|c|c|c|c|}
\hline Sample & Specimen nr. & Species & $\begin{array}{l}\text { Tooth } \\
\text { position }\end{array}$ & $\begin{array}{l}\delta^{13} \mathrm{C} \\
\text { VPDB } \\
(\%)\end{array}$ & SD & $\begin{array}{l}\delta^{18} \mathrm{O} \\
\text { VPDB } \\
(\% \text { ) }\end{array}$ & SD & $\begin{array}{l}\delta^{18} \mathrm{O} \\
\text { VSMOW } \\
(\%)\end{array}$ & $\begin{array}{l}\mathrm{CaCO}_{3} \\
\text { (wt. } \% \text { ) }\end{array}$ \\
\hline FZ SU SA 3 & BSPG 195911265 & Hyotherium soemmeringi & $\mathrm{p}$ & -11.1 & 0.05 & -7.8 & 0.11 & 22.8 & 5.1 \\
\hline FZ SU SA 4 & BSPG 195911265 & Hyotherium soemmeringi & $\mathrm{p} 4$ & -11.2 & 0.06 & -8.1 & 0.08 & 22.6 & 5.6 \\
\hline FZ SU SA 5 & BSPG 195911266 & Hyotherium soemmeringi & M & -10.1 & 0.06 & -8.6 & 0.10 & 22.1 & 4.3 \\
\hline FZ SU SA 6 & BSPG 195911266 & Hyotherium soemmeringi & M & -9.9 & 0.06 & -9.0 & 0.09 & 21.6 & 4.4 \\
\hline FZ SU SA 7 & No nr. & Hyotherium soemmeringi & $\mathrm{P}$ & -9.2 & 0.06 & -6.7 & 0.13 & 24.0 & 4.7 \\
\hline FZ SU SA 8 & No nr. & Hyotherium soemmeringi & I & -9.6 & 0.07 & -8.7 & 0.12 & 21.9 & 5.3 \\
\hline
\end{tabular}

Table 2 Mean enamel $\delta^{13} \mathrm{C}$ and $\delta^{18} \mathrm{O}$ values

\begin{tabular}{|c|c|c|c|c|c|c|c|c|}
\hline Species & Skeletal tissue & $n$ & $\begin{array}{l}\delta^{13} \mathrm{C} \\
\text { VPDB } \\
(\%)\end{array}$ & SD & Range & $\begin{array}{l}\delta^{18} \mathrm{O} \\
\text { VPDB } \\
(\%)\end{array}$ & SD & Range $(\%)$ \\
\hline Germanomeryx fahlbuschi & Enamel & 3 & -12.0 & 0.8 & -12.6 to -11.1 & -5.7 & 1.7 & -7.7 to -4.5 \\
\hline Metaschizotherium sp. & Enamel & 5 & -12.7 & 0.8 & -13.9 to -11.9 & -7.5 & 0.8 & -8.3 to -6.3 \\
\hline Prosantorhinus germanicus & Enamel & 6 & -12.4 & 0.7 & -13.6 to -11.7 & -7.2 & 0.7 & -8.1 to -6.0 \\
\hline Plesiaceratherium fahlbuschi & Enamel & 8 & -11.6 & 0.5 & -12.5 to -11.0 & -7.2 & 0.7 & -7.8 to -6.0 \\
\hline Lartetotherium sansaniense & Enamel & 4 & -11.0 & 0.7 & -11.6 to -10.2 & -7.3 & 0.6 & -7.8 to -6.5 \\
\hline Gomphotherium subtapiroideum & Enamel & 7 & -11.1 & 0.6 & -11.9 to -10.2 & -8.1 & 1.0 & -9.6 to -6.8 \\
\hline Heteroprox eggeri & Enamel & 4 & -11.0 & 0.7 & -12.0 to -10.4 & -8.1 & 1.6 & -9.4 to -6.0 \\
\hline Anchitherium aurelianense & Enamel & 8 & -11.1 & 0.7 & -11.8 to -9.5 & -6.6 & 0.9 & -7.9 to -5.5 \\
\hline Hyotherium soemmeringi & Enamel & 8 & -10.2 & 0.7 & -11.2 to -9.2 & -8.4 & 0.9 & -9.8 to -6.7 \\
\hline
\end{tabular}

spectrometer at the University of Lausanne. For this reaction an acid fractionation factor of 1.008818, the same as between calcite and $\mathrm{CO}_{2}$, was assumed to be applicable. The measured carbon and oxygen isotopic compositions were normalized to the in-house Carrara marble calcite standard that has been calibrated against the international NBS-19 calcite standard. The isotope composition of tooth enamel apatite is reported in the usual $\delta$-notation in permille (\%o) relative to the known isotope reference standard Vienna Pee Dee Belemnite (VPDB) (Coplen 1994).

$\delta^{13} \mathrm{C}$ or $\delta^{18} \mathrm{O}(\%)=\left[\left(R_{\text {sample }} / R_{\text {standard }}\right)-1\right] \times 1,000$,

where $R_{\text {sample }}$ and $R_{\text {standard }}$ are the ${ }^{13} \mathrm{C} /{ }^{12} \mathrm{C}$ and ${ }^{18} \mathrm{O} /{ }^{16} \mathrm{O}$ ratios in the sample and standard, respectively. Precision for the carbon $\left(\delta^{13} \mathrm{C}\right)$ and oxygen $\left(\delta^{18} \mathrm{O}\right)$ isotopic composition of carbonate in the apatite is better than $\pm 0.1 \%$ and $\pm 0.15 \%$, respectively. The NBS 120c Florida phosphate rock standard, also pretreated after Koch et al. (1997), gave values of $\delta^{13} \mathrm{C}_{\mathrm{VPDB}}=-6.3 \pm 0.1 \%$ and $\delta^{18} \mathrm{O}_{\mathrm{VPDB}}=$ $-2.3 \pm 0.2 \%$ o $(n=13)$.

\section{$O$ isotope measurements of the phosphate}

The oxygen isotope composition of phosphate $\left(\delta^{18} \mathrm{O}_{\mathrm{PO}_{4}}\right)$ was measured on silver phosphate $\left(\mathrm{Ag}_{3} \mathrm{PO}_{4}\right)$ precipitated according to a method modified after Dettmann et al. (2001) and described in Tütken et al. (2006). Four milligrams pretreated enamel powder was dissolved in $2 \mathrm{M} \mathrm{HF}$ in a 2-ml safe-lock centrifuge vessel. After centrifuging, the HF solution was transferred to a new centrifuge vessel, leaving the $\mathrm{CaF}_{2}$ residue behind. After neutralization with $25 \% \mathrm{NH}_{4} \mathrm{OH}$ the dissolved phosphate was precipitated as $\mathrm{Ag}_{3} \mathrm{PO}_{4}$ by addition of $2 \mathrm{M} \mathrm{AgNO} 3$ solution. $\mathrm{Ag}_{3} \mathrm{PO}_{4}$ of each sample was analyzed in triplicate for its oxygen isotopic composition according to methods described in Vennemann et al. (2002) using a TC-EA at $1,450^{\circ} \mathrm{C}$, linked to a ThermoFinnigan Delta Plus XL gas mass spectrometer at the University of Lausanne. $\delta^{18} \mathrm{O}_{\mathrm{PO}_{4}}$ values are reported in the usual $\delta$-notation versus VSMOW. The $\mathrm{Ag}_{3} \mathrm{PO}_{4}$ precipitated from the NBS 120c standard gave a mean $\delta^{18} \mathrm{O}_{\mathrm{PO}_{4}}$ value of $21.6 \pm 0.4 \%$ o $(n=25)$.

\section{Sr isotope measurements}

The preparation for $\mathrm{Sr}$ isotope analysis was done in a clean laboratory. A $1 \mathrm{mg}$ aliquot of the pretreated enamel powder was dissolved in $1 \mathrm{ml}$ suprapure $\mathrm{HNO}_{3}$. The $\mathrm{Sr}$ fraction was separated with a standard separation procedure on quartz glass columns filled with $5 \mathrm{ml}$ cation exchange resin bed of BioRad AG 50W-X12, 200-400 mesh. The purified 
Table $3 \delta^{13} \mathrm{C}$ and $\delta^{18} \mathrm{O}$ values of serial sampled Gomphotherium tusk enamel

\begin{tabular}{lllllll}
\hline Sample & $\begin{array}{l}\text { Distance } \\
(\mathrm{mm})\end{array}$ & $\begin{array}{l}\delta^{18} \mathrm{O} \\
\text { VPDB } \\
(\%)\end{array}$ & $\begin{array}{l}\mathrm{SD} \\
\delta^{13} \mathrm{C} \\
\text { VPDB } \\
(\%)\end{array}$ & $\mathrm{SD}$ & $\begin{array}{l}\mathrm{CaCO}_{3} \\
(\mathrm{wt} \%)\end{array}$ \\
\hline FZ MA SA 3-1 & 1 & -9.0 & 0.3 & -12.0 & 0.2 & 3.8 \\
FZ MA SA 3-2 & 3 & -9.0 & 0.2 & -12.1 & 0.2 & 4.5 \\
FZ MA SA 3-3 & 5 & -9.4 & 0.2 & -12.0 & 0.1 & 4.2 \\
FZ MA SA 3-4 & 9 & -10.3 & 0.3 & -11.9 & 0.2 & 3.7 \\
FZ MA SA 3-5 & 12 & -9.7 & 0.2 & -12.0 & 0.1 & 3.8 \\
FZ MA SA 3-6 & 15 & -10.1 & 0.2 & -12.2 & 0.1 & 3.6 \\
FZ MA SA 3-7 & 19 & -9.6 & 0.3 & -11.8 & 0.1 & 3.9 \\
FZ MA SA 3-8 & 25 & -9.3 & 0.2 & -12.2 & 0.1 & 4.2 \\
FZ MA SA 3-9 & 28 & -9.6 & 0.2 & -11.8 & 0.2 & 4.1 \\
FZ MA SA 3-10 & 32 & -9.9 & 0.2 & -12.0 & 0.1 & 4.3 \\
FZ MA SA 3-11 & 36 & -10.2 & 0.2 & -11.9 & 0.2 & 3.8 \\
FZ MA SA 3-12 & 40 & -9.4 & 0.2 & -12.1 & 0.1 & 4.0 \\
FZ MA SA 3-13 & 45 & -10.8 & 0.2 & -11.9 & 0.1 & 3.9 \\
FZ MA SA 3-14 & 50 & -9.2 & 0.1 & -11.7 & 0.1 & 4.8 \\
FZ MA SA 3-15 & 54 & -9.7 & 0.2 & -11.7 & 0.1 & 4.4 \\
FZ MA SA 3-16 & 58 & -9.3 & 0.1 & -11.9 & 0.1 & 4.7 \\
\hline
\end{tabular}

Table 4 Enamel $\delta^{18} \mathrm{O}_{\mathrm{PO}_{4}}$ values and calculated drinking water $\delta^{18} \mathrm{O}_{\mathrm{H}_{2} \mathrm{O}}$ and MAT values

\begin{tabular}{llllll}
\hline Sample & $\begin{array}{l}\delta^{18} \mathrm{OPO}_{4} \\
\text { VSMOW } \\
(\%)\end{array}$ & $\mathrm{SD}$ & $n$ & $\begin{array}{l}\delta^{18} \mathrm{O}_{\mathrm{H}_{2} \mathrm{O}} \\
\text { VSMOW } \\
(\%)\end{array}$ & $\begin{array}{l}\mathrm{MAT} \\
\left({ }^{\circ} \mathrm{C}\right)\end{array}$ \\
\hline FZ EQ SA 1 & 19.0 & 0.4 & 3 & -5.6 & 19.3 \\
FZ EQ SA 2 & 19.1 & 0.3 & 3 & -5.5 & 19.6 \\
FZ EQ SA 4 & 19.6 & 0.3 & 3 & -4.8 & 21.2 \\
FZ MA SA 4 & 17.9 & 0.3 & 3 & -5.8 & 19.1 \\
FZ RH SA 1 & 18.2 & 0.5 & 3 & -5.3 & 20.1 \\
FZ RH SA 4 & 18.1 & 0.3 & 3 & -5.4 & 19.8 \\
FZ RH SA 7a & 15.9 & 0.3 & 3 & -7.0 & 16.2 \\
\hline
\end{tabular}

Table 5 Strontium isotope composition of tooth enamel

\begin{tabular}{llll}
\hline Sample & Species & ${ }^{87} \mathrm{Sr} /{ }^{86} \mathrm{Sr}$ & $2 \sigma$ \\
\hline FZ EQ SA 1 & Anchitherium aurelianense & 0.710319 & 0.000009 \\
FZ MA SA 3 & Gomphotherium subtapiroideum & 0.710628 & 0.000009 \\
FZ MA SA 4 & Gomphotherium subtapiroideum & 0.710427 & 0.000010 \\
FZ MA SA 5 & Gomphotherium subtapiroideum & 0.710154 & 0.000010 \\
\hline
\end{tabular}

$\mathrm{Sr}$ was loaded on tungsten filaments coated with $\mathrm{TaF}_{5}$ activator. The $\mathrm{Sr}$ isotopic composition was measured with a Finnigan MAT 262 thermal ionization mass spectrometer (TIMS) at the University of Tübingen. For each sample $>200{ }^{87} \mathrm{Sr} /{ }^{86} \mathrm{Sr}$ ratios were measured in the static mode

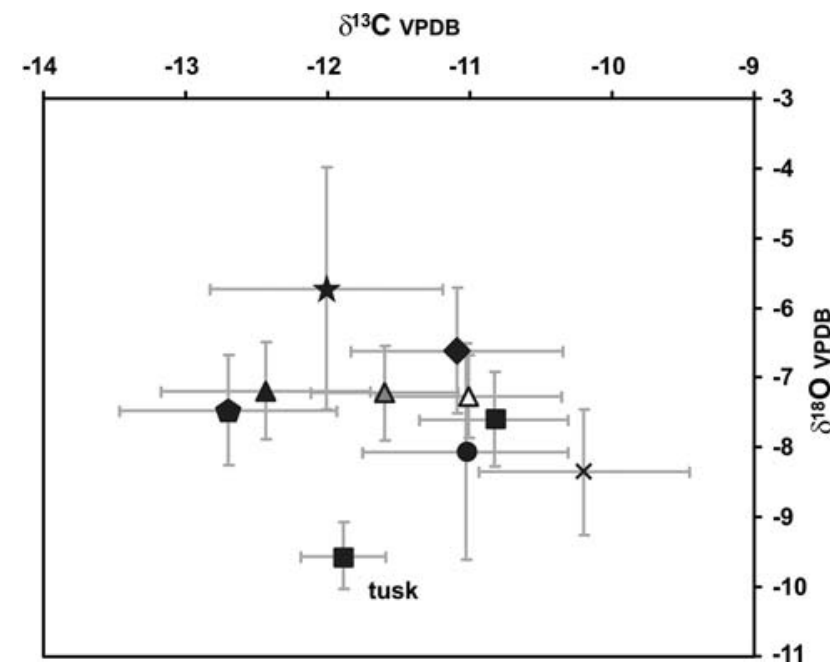

Fig. 2 Enamel carbonate mean $\delta^{13} \mathrm{C}$ and $\delta^{18} \mathrm{O}$ values and one standard deviations of the nine analyzed mammal taxa from Sandelzhausen: Metaschizotherium bavaricum (pentagon), the three rhinoceroses Prosantorhinus germanicus (filled triangle), Plesiaceratherium fahlbuschi (shaded triangle), and Lartetotherium sansaniense (open triangle), the equid Anchitherium aurelianense (filled diamond), the cervid Heteroprox eggeri (filled circle), the palaeomerycid Germanomeryx fahlbuschi (star), the suid Hyotherium soemmeringi (cross), and the proboscidean Gomphotherium subtapiroideum (filled square). For the mean value of Gomphotherium the D4 tooth and tusk were excluded for reasons explained in the text. The mean value of the data from the tusk sample (Table 3) is shown separately. Analytical error is about the symbol size

with internal precision $\leq 10 \times 10^{-6} \cdot{ }^{87} \mathrm{Sr} /{ }^{86} \mathrm{Sr}$ ratios were corrected for mass fractionation in the instrument using the natural ${ }^{88} \mathrm{Sr} /{ }^{86} \mathrm{Sr}$ ratio of 8.375209 . Measured ${ }^{87} \mathrm{Sr} /{ }^{86} \mathrm{Sr}$ ratios were normalized to the certified value of NBS 987 $\left({ }^{87} \mathrm{Sr} /{ }^{86} \mathrm{Sr}=0.710248\right)$. The NBS 987 gave a mean ${ }^{87} \mathrm{Sr} /{ }^{86} \mathrm{Sr}=0.710246 \pm 9(n=7)$, during the period of $\mathrm{Sr}$ isotope measurements.

\section{Results}

Enamel $\delta^{13} \mathrm{C}$ values

The enamel $\delta^{13} \mathrm{C}$ values of the 53 individual teeth analyzed have a range from $-9.2 \%$ to $-13.9 \%$ (Fig. 2, Table 1) and a mean value of $-11.4 \pm 1.0 \%$. Mean $\delta^{13} \mathrm{C}$ values of the different taxa range from $-10.2 \%$ to $-12.7 \%$ (Table 2). The chalicothere M. bavaricum $(-12.7 \pm 0.8 \%$ ) has the lowest mean $\delta^{13} \mathrm{C}$ value of all analyzed large mammal taxa from Sandelzhausen. The rhinoceros P. germanicus $(-12.4 \pm 0.7 \%)$ and the palaeomerycid G. fahlbuschi $\left(-12.0 \pm 0.8 \%\right.$ ) also had similar low $\delta^{13} \mathrm{C}$ values. The three sympatric rhinoceros species have different mean $\delta^{13} \mathrm{C}$ values: $P$. germanicus $(-12.4 \pm 0.7 \%)$, the lowest value of the three sympatric rhinoceros species, P. fahlbuschi 
$(-11.6 \pm 0.5 \%)$ with intermediate values, and $L$. sansaniense $(-11.0 \pm 0.7 \%$ ) with the highest values. Most other large mammals, such as A. aurelianense (-11.1 $\pm 0.7 \%$ ), G. subtapiroideum $(-11.1 \pm 0.6 \%)$, and H. eggeri $\left(-11.0 \pm 0.7 \%\right.$ ) have identical mean $\delta^{13} \mathrm{C}$ values of around $-11 \%$, only slightly more positive than the mean average value for the site. The G. subtapiroideum tusk has a significantly lower mean $\delta^{13} \mathrm{C}$ value of $-11.9 \pm 0.1 \%$ o $(n=16)$ that is lower than that of the the molars (Fig. 2, Table 3). Furthermore, the analyzed zones of this tusk have a $\Delta \delta^{13} \mathrm{C}$ value $\left(\Delta \delta^{13} \mathrm{C}\right.$ is the difference between the lowest and highest $\delta^{13} \mathrm{C}$ value) of only $0.5 \%$. The suid $H$. soemmeringi $(-10.2 \pm 0.7 \%$ o $)$ has the highest mean $\delta^{13} \mathrm{C}$ value of all taxa analyzed from Sandelzhausen, which is about $1 \%$ higher than the mean value for all taxa (Fig. 2). Most mammals have a similar intrataxon $\delta^{13} \mathrm{C}$ variability, $\Delta \delta^{13} \mathrm{C}$, of around 2\%o (Fig. 3). However, the large rhinoceros L. sansaniense has the smallest $\Delta \delta^{13} \mathrm{C}$ value of $1.4 \%$ and $A$. aurelianense has the highest $\Delta \delta^{13} \mathrm{C}$ value of $2.3 \%$ of all taxa (Fig. 3).

Enamel $\delta^{18} \mathrm{O}_{\mathrm{CO}_{3}}$ values

The $\delta^{18} \mathrm{O}$ values of the mammal teeth display a range from $-4.5 \%$ to $-9.8 \%$ (Table 1 ) and a mean value of $-7.4 \pm 1.1 \%$ o $(n=53)$. The suid H. soemmeringi $\left(-8.4 \pm 0.9 \%\right.$ o has the lowest mean $\delta^{18} \mathrm{O}$ value of all large mammal taxa from Sandelzhausen analyzed. G. subtapiroideum $(-8.1 \pm 1.0 \%$ ) and $H$. eggeri $(-8.1 \pm 1.6 \%$ )

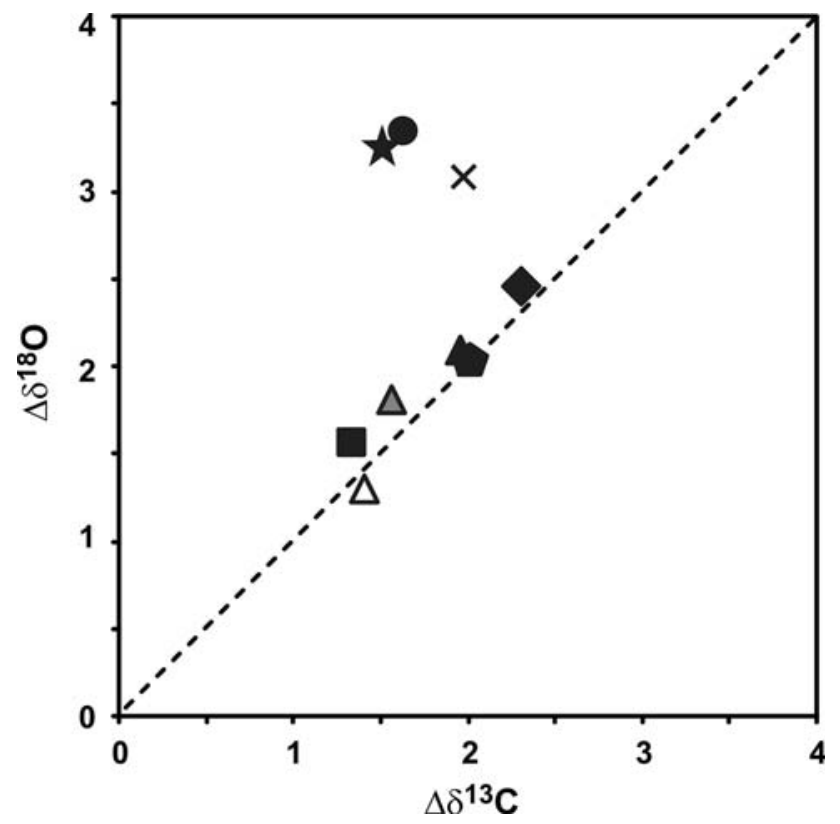

Fig. 3 Intrataxon ranges of enamel carbon $\left(\Delta \delta^{13} C\right)$ and oxygen $\left(\Delta \delta^{18} \mathrm{O}\right)$ compositions of the nine analyzed mammal taxa from Sandelzhausen. Same symbols as in Fig. 2. For Gomphotherium the D4 tooth and the tusk were excluded for reasons explained in the text have similar low mean $\delta^{18} \mathrm{O}$ values. Most other mammals have higher mean $\delta^{18} \mathrm{O}$ values between $-7 \%$ and $-7.5 \%$ (Fig. 2, Table 2). The three sympatric rhinoceros species have identical mean $\delta^{18} \mathrm{O}$ values: $P$. germanicus $(-7.2 \pm 0.7 \%$ ),$\quad$ P. fahlbuschi $\quad(-7.2 \pm 0.7 \%$ ), and L. sansaniense $(-7.3 \pm 0.6 \%)$. Only slightly lower is the mean $\delta^{18} \mathrm{O}$ value of $M$. bavaricum $(-7.5 \pm 0.8 \%$ ). A. aurelianense $\left(-6.6 \pm 0.9 \%\right.$ ) has a slightly higher $\delta^{18} \mathrm{O}$ value. The palaeomerycid G. fahlbuschi $(-5.7 \pm 1.7 \%$ ) has the highest mean and single $\delta^{18} \mathrm{O}$ value of all analyzed taxa (Fig. 3). Furthermore, G. fahlbuschi and H. eggeri also have the highest variability of $\delta^{18} \mathrm{O}$ values expressed with a $\Delta \delta^{18} \mathrm{O}$ value $\left(\Delta \delta^{18} \mathrm{O}\right.$ is the difference between the lowest and highest $\delta^{18} \mathrm{O}$ value) of $3 \%$ (Fig. 3 ). The Gomphotherium suptapiroideum tusk has a mean $\delta^{18} \mathrm{O}$ value of $-9.7 \pm 0.5 \%$ o $(n=16)$, lower than any other mammal teeth (Fig. 2, Table 3). The intratusk $\Delta \delta^{18} \mathrm{O}$ range is $1.8 \%$ (Fig. 4).

Enamel $\delta^{18} \mathrm{O}_{\mathrm{PO}_{4}}$ values

The enamel of seven molar teeth has been analyzed for the phosphate oxygen isotope composition of the apatite. Enamel $\delta{ }^{18} \mathrm{O}_{\mathrm{PO}_{4}}$ VSMOW values for the Anchitherium range from $19.9 \%$ to $19.6 \%$ o $(n=3)$, those for the rhinoceros from $15.9 \%$ to $18.2 \% \quad(n=3)$, and one Gomphotherium molar has a value of $17.9 \%$ (Table 4 ). These $\delta^{18} \mathrm{O}$ values indicate that the animals used water sources with $\delta^{18} \mathrm{O}_{\mathrm{H}_{2} \mathrm{O}}$ values of $-4.8 \%$ to $-7.0 \%$ VSMOW (Table 4).

\section{Sr isotope composition}

The enamel ${ }^{87} \mathrm{Sr} /{ }^{86} \mathrm{Sr}$ ratios of the Anchitherium tooth (0.71033) and the Gomphotherium teeth (0.71013-0.7106) are similar and range from 0.71013 to 0.71063 (Table 5). The ${ }^{87} \mathrm{Sr} /{ }^{86} \mathrm{Sr}$ ratios are higher than those of teeth from sympatric Miocene mammals of the Molasse Basin and surrounding areas (Tütken et al. 2006; Fig. 5).

\section{Discussion}

Despite the small overall range of isotope compositions $\left(\delta^{13} \mathrm{C}=-9.2 \%\right.$ to $-13.9 \%$ and $\delta^{18} \mathrm{O}=-4.5 \%$ to $-9.8 \%$ ) some of the nine analyzed large mammal taxa showed significant differences in their enamel carbon and oxygen isotope composition (Fig. 2). Although most skeletal remains from Sandelzhausen were disarticulated, most lack transport marks, so that transport over long distances and thus potential mixing of bones and teeth of animals from ecologically distinct habitats is unlikely. Furthermore, the intertaxon isotope differences are not due to significant time 
Fig. 4 Carbon (filled diamond) and oxygen (open triangle) isotope data from the serially sampled Gomphotherium subtapiroideum tusk (sample FZ MA SA 3 from specimen BSPG 1959 II 44, Table 3). The stippled rectangle in the inset photograph shows the part of the tusk from which the 16 samples were taken

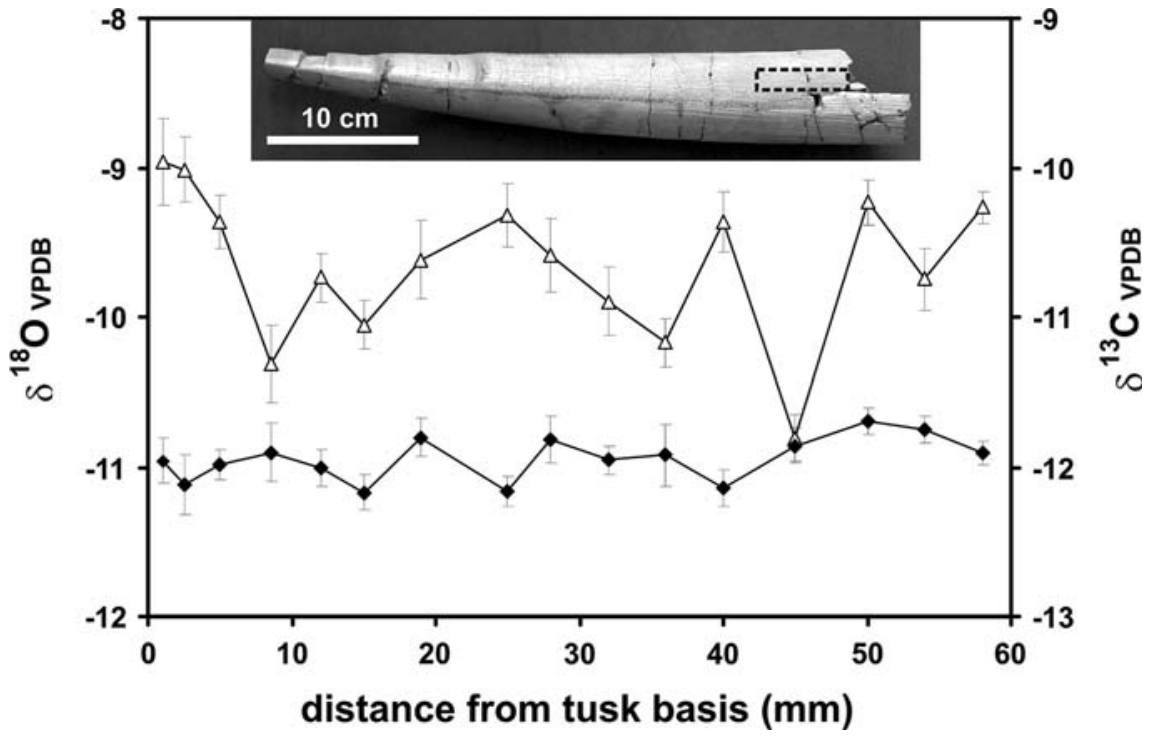

Fig. $5 \mathrm{Sr}$ isotope compositions of enamel from fossil mammal teeth from Miocene localities in southern Germany and Switzerland. Filled triangle Sandelzhausen, this study; shaded triangle ${ }^{87} \mathrm{Sr} /{ }^{86} \mathrm{Sr}$ data from Tütken et al. (2006) and Tütken unpublished data: $C H$ Charmoille, Switzerland; $B E$ Benken, Switzerland; $L A$ Ulm Langenau, SW Germany; $H E$ Helsighausen, Switzerland; $K \ddot{A}$ Käpfnach, Switzerland; $H \ddot{U}$ Hülistein, Switzerland; $E N$ Engelswies, SW Germany; ST Steinheim, Swabian Alb, SW Germany; $N \ddot{O}$ Nördlinger Ries, Swabian Alb, S Germany; $H W$ Höwenegg, Hegau, SW Germany

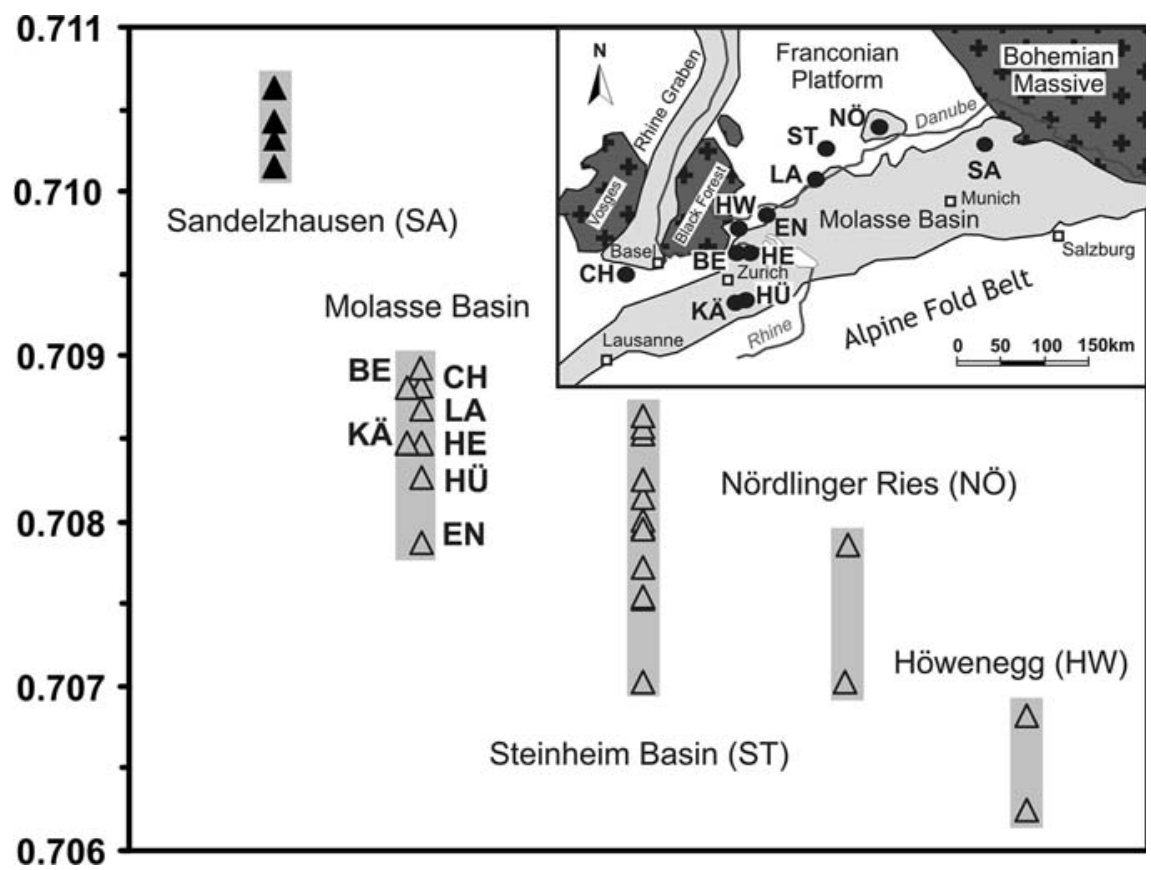

averaging because of the short deposition time of the sediment succession at Sandelzhausen (Moser et al. 2009b, this issue). This is further supported by the fact that enamel samples of teeth from the same taxon in the lower and upper part of the sediment profile, which are separated by a thin coaly layer, do not have taxonwise differences in their carbon and oxygen isotope compositions. Thus, no significant changes in diet and/or habitat for the analyzed taxa are associated with the evolution from a more fluvial to a limnic depositional environment. Therefore, enamel samples from both stratigraphic units are pooled for each taxon for the following discussion of the data. The observed intertaxon differences in isotope compositions can be attributed to dietary, environmental or physiological differences such as different feeding ecology, habitat use or digestive strategy.

Carbon isotopes: general consideration

The teeth from all large herbivores sampled from Sandelzhausen have a mean enamel $\delta^{13} \mathrm{C}$ value of $-11.4 \pm$ $0.7 \%$ and a range from $-9.2 \%$ to $-13.9 \%$, clearly indicating that all herbivores fed on $\mathrm{C}_{3}$ plants only. The range of enamel $\delta^{13} \mathrm{C}$ values is comparable to that of large herbivorous mammals from other Miocene $\mathrm{C}_{3}$-plant-dominated settings, e.g., Ternan, East Africa (14 Ma): $-8.6 \%$ to $-13 \%$ (Cerling et al. 1997a); Panama (15 Ma): $-10.1 \%$ to 
$-15.9 \%$ (MacFadden and Higgins 2004), and the Siwaliks, Pakistan (9.2 Ma): $-8.1 \%$ to $-14.6 \%$ (Nelson 2007). In such a pure $\mathrm{C}_{3}$ plant ecosystem the intertaxon differences in enamel $\delta^{13} \mathrm{C}$ values (Fig. 2) are related to feeding on $\mathrm{C}_{3}$ plants with distinct $\delta^{13} \mathrm{C}$ values which are related to differences in habitat (e.g., vegetation openness, humidity, biomass recycling; Ehleringer et al. 1986, 1987; van der Merwe and Medina 1989) and/or plant tissues (e.g., leaves, stems, fruits; Heaton 1999; Cerling et al. 2004). The enamel $\delta{ }^{13} \mathrm{C}$ values of all Sandelzhausen mammals are well in accordance with foraging in forests and open woodlands.

However, different digestive physiology, especially the rate of methane formation, can also cause intertaxon differences in enamel $\delta^{13} \mathrm{C}$ values (Passey et al. 2005). Passey et al. (2005) found a total range of 5\% for enamel $\delta^{13} \mathrm{C}$ values of different small and large mammals raised on a controlled diet with the same carbon isotopic composition. However, for large ruminant and nonruminant herbivorous mammals they only found a difference of around $1.3 \%$. Intertaxon $\delta^{13} \mathrm{C}$ differences larger than that must be caused by other factors. For extinct mammals such as metaschizotheres or palaeomerycids, which have the most distinct isotope compositions of all Sandelzhausen mammals (Fig. 2), the digestive strategy is unknown, hence its effect on enamel $\delta^{13} \mathrm{C}$ values cannot be determined. However, for Sandelzhausen mammals, a systematic difference in enamel $\delta^{13} \mathrm{C}$ values exists even between species using the same digestive physiology, especially between the three sympatric rhinoceros species (Fig. 2), which are all hindgut fermenters. This supports dietary and/or habitat differences being a major factor controlling enamel $\delta^{13} \mathrm{C}$ values of the mammal foraging in the $C_{3}$ plant ecosystem of Sandelzhausen.

None of the enamel $\delta^{13} \mathrm{C}$ values from Sandelzhausen are consistent with a diet derived from closed-canopy conditions. In such environments with dense forest cover, low light intensities, and $\mathrm{CO}_{2}$ recycling (van der Merwe and Medina 1989), herbivores have enamel values lower than $-14 \%$ to $15 \%$ (Cerling et al. 2004; Kohn et al. 2005). However, enamel $\delta^{13} \mathrm{C}$ values of $\leq-12 \%$ and lower for Metaschizotherium, Prosantorhinus, and Germanomeryx (Fig. 2) suggest feeding in a closed forest or woodland habitat, relative to the other sympatric mammals. Most taxa, such as Gomphotherium, Lartetotherium, Anchitherium, and Heteroprox have very similar mean enamel $\delta^{13} \mathrm{C}$ values of around $-11 \%$ (Fig. 2), as expected for mammals feeding on average Miocene $\mathrm{C}_{3}$ plants with a $\delta^{13} \mathrm{C}$ value of around $-25 \%$. These taxa were probably nonselective feeders and did not feed on certain food plants and/or in closed or open environments only. The forest hog Hyotherium has the highest mean $\delta^{13} \mathrm{C}$ value $(-10.2 \%$ ) and thus ingested the most ${ }^{13} \mathrm{C}$-rich diet of all taxa from Sandelzhausen and possibly was omnivorous.
Oxygen isotopes: general consideration

The palaeomerycid G. fahlbuschi has the highest mean $\delta^{18} \mathrm{O}$ value $(-5.7 \%$ ) and, together with the cervid $H$. eggeri, also the largest range of $\delta^{18} \mathrm{O}$ values $\left(\Delta \delta^{18} \mathrm{O}=3.2 \%\right.$ ) of all taxa (Fig. 3). Probably Germanomeryx foraged preferentially on leaves from the canopy that are ${ }^{18} \mathrm{O}$ enriched. Most other taxa have mean enamel $\delta^{18} \mathrm{O}$ values around $-7.5 \pm 0.3 \%$ similar to the locality mean of $-7.4 \%$. Only the Anchitherium has a slightly higher mean $\delta^{18} \mathrm{O}$ value of $-6.6 \%$, possibly related to feeding on a somewhat more ${ }^{18} \mathrm{O}$-enriched diet and/or feeding in a more open environment. In contrast, H. eggeri $(-8 \%$ ) and especially the forest hog Hyotherium $(-8.4 \%$ ) have the lowest mean $\delta^{18} \mathrm{O}$ values of all taxa. They probably fed in a more humid environment and/or were more water dependent. Water-dependent species track the $\delta^{18} \mathrm{O}$ value of meteoric water (Longinelli 1984; Kohn 1996; Levin et al. 2006). Most species in Sandelzhausen have $\delta^{18} \mathrm{O}$ values expected for obligate drinkers and the mean $\delta^{18} \mathrm{O}$ value is similar to those of large mammals from other Miocene localities in southern Germany (Tütken et al. 2006).

The four teeth analyzed for their phosphate oxygen isotope composition $\left(\delta^{18} \mathrm{O}_{\mathrm{PO}_{4}}\right)$ allow a mean meteoric water $\delta^{18} \mathrm{O}_{\mathrm{H}_{2} \mathrm{O}}$ value of $-5.6 \pm 0.7 \%$ VSMOW to be calculated, given their species-specific $\delta^{18} \mathrm{O}_{\mathrm{PO}_{4}}-\delta^{18} \mathrm{O}_{\mathrm{H}_{2} \mathrm{O}}$ relations for modern horses (Huertas et al. 1995), elephants (Ayliffe et al. 1992), and rhinoceroses (Tütken et al. 2006). This value provides an estimate of a mean $\delta^{18} \mathrm{O}$ value for the late Early/early Middle Miocene meteoric water and is similar to those reconstructed for other Miocene localities of Germany (Tütken et al. 2006, Tütken unpublished data). Using a MAT $-\delta^{18} \mathrm{O}_{\mathrm{H}_{2} \mathrm{O}}$ regression based on modern precipitation and air temperature data for Germany and Switzerland (see Tütken et al. 2006 for details), a mean MAT of $19.3 \pm 1.5^{\circ} \mathrm{C}$ can be calculated for Sandelzhausen (Table 4), which is about $10^{\circ} \mathrm{C}$ warmer than today. Due to the small sample size this derived MAT has to be considered with caution, but it is reasonable because the Middle Miocene was the warmest period of the Neogene (Zachos et al. 2001). Furthermore, it is in good agreement with a MAT estimate ranging from $18^{\circ} \mathrm{C}$ to $20.8^{\circ} \mathrm{C}$ based on the occurrence of ectothermic vertebrates in Sandelzhausen and southern Germany (Böhme 2009, this issue).

In the following the isotopic results for each of the nine investigated mammal taxa from Sandelzhausen will be discussed in more detail and in the context of known information about their diet.

Diet of the chalicothere $M$. bavaricum

Metaschizotherium bavaricum, the extinct, large, clawbearing Perissodactyl, is a rare faunal element in the 
Sandelzhausen fauna. The occurrence of Metaschizotherium indicates the presence of trees, although not necessarily with dense tree cover. Metaschizotheres were probably bipedal brachydont browsers mostly interpreted to be leafeating herbivores (e.g., Heissig 1999). However, their diet is still a matter of debate. Recent mesowear results suggest that $M$. bavaricum was a browser that fed on both nonabrasive plants but also abrasive fibrous and tough plant material such as bark, twigs, and branches likely in a closed, nondusty forest environment (Schulz et al. 2007; Coombs 2009, this issue; Schulz and Fahlke 2009, this issue). Feeding in a more closed, forested environment is well in accordance with the lowest mean $(-12.7 \%)$ as well as the lowest single enamel $\delta^{13} \mathrm{C}$ value $(-13.9 \%$ ) for Metaschizotherium of all Sandelzhausen mammals. However, this value was obtained from a first molar (M1). Assuming a similar dental development strategy as for other large mammals, this value might be influenced at least to some extent by the maternal milk composition as the M1 mineralizes after birth and mostly before weaning. However, even excluding the M1, the mean value $(-12.4 \%$ ) is still low. Only the small rhinoceros $P$. germanicus has a similarly low mean $\delta^{13} \mathrm{C}$ value (Fig. 2). Such a low mean $\delta^{13} \mathrm{C}$ value of below $-12 \%$ also supports feeding in a forested woodland (e.g., Passey et al. 2002), but the values are not as low as would be expected from forest-dwelling subcanopy browsers in a closed-canopy environment (Cerling et al. 2004). Within a modern rainforest canopy plants growing in gaps of the canopy or leaves, fruits, and seeds from the canopy have about 4-5\%o higher $\delta^{13} \mathrm{C}$ values compared with subcanopy plants (van der Merwe and Medina 1989; Cerling et al. 2004). Therefore, subcanopy frugivores and folivores as well as omnivores living in open areas within the forest have higher $\delta^{13} \mathrm{C}$ values compared with subcanopy browsers (Cerling et al. 2004). The magnitude of such canopy effects on plant $\delta^{13} \mathrm{C}$ values is much less developed in sparsely forested realms. However, still Metaschizotherium was probably not using fruits and seeds from the canopy as a major food source because higher enamel $\delta^{13} \mathrm{C}$ and especially $\delta^{18} \mathrm{O}$ values would be expected as fruits and seeds tend to be enriched in ${ }^{18} \mathrm{O}$ relative to the source water, similar to leaves (Yakir 1992, 1997). However, Metaschizotherium has enamel $\delta^{18} \mathrm{O}$ values similar to most other large mammals and not such high values as the sympatric palaeomerycid G. fahlbuschi, which was probably a canopy folivore.

Diet of the rhinoceroses

Rhinoceros are the most abundant large mammals in the Sandelzhausen fauna and there were three sympatric species: P. germanicus, P. fahlbuschi, and L. sansaniense
(Heissig 1972; Peter 2002). These rhinoceroses are all relatively well adapted to moist, swampy habitat and indicate a water-rich environment (Heissig 2005, personal communication). P. germanicus is the smallest and most frequent of the three species. It has short legs and mesodont cheek teeth adapted to a somewhat abrasive diet, possibly reeds or other abrasive plants in a swampy environment (Heissig 2005, personal communication). P. fahlbuschi is a medium-sized species. L. sansaniense is the least frequent and largest rhinoceros species present in Sandelzhausen. It has low-crowned cheek teeth and likely fed on a soft diet (Heissig 2005, personal communication). Lartetotherium was probably a less specialized feeder occurring in different habitats and was a long-lived species (Heissig 1972).

All three rhinoceros taxa have different mean enamel $\delta{ }^{13} \mathrm{C}$ values (Fig. 2), especially the two most abundant species, Prosantorhinus and Plesiaceratherium, which have $1 \%$ difference in their mean enamel $\delta^{13} \mathrm{C}$ values. This indicates the use of isotopically different food plants due to niche partitioning and/or habitat differences of the sympatric rhinoceros. Prosantorhinus has the lowest $\delta^{13} \mathrm{C}$ value and could well have been a browser in a partly more closed, forested habitat. Plesiaceratherium has an intermediate and Lartetotherium the highest mean enamel $\delta^{13} \mathrm{C}$ value, hence Lartetotherium was probably feeding in a more open environment than the other two species.

All three rhinoceros species seem to have been waterdependent species and probably obligate drinkers as they have identical enamel $\delta^{18} \mathrm{O}$ values of $-7.3 \%$ similar to the locality mean (Fig. 2). The three rhinoceros species plot distinct from each other in the $\Delta \delta^{18} \mathrm{O}-\Delta \delta^{13} \mathrm{C}$ diagram (Fig. 3). Together with the slightly different mean enamel $\delta^{13} \mathrm{C}$ values this supports niche partitioning of the sympatric rhinoceros. Higher $\Delta \delta^{13} \mathrm{C}$ and $\Delta \delta^{18} \mathrm{O}$ values indicate the use of different food sources with variable isotope compositions. If this is true, then Prosantorhinus would have been the more flexible or generalistic feeder and Lartetotherium a more specialized feeder. However, this seems to be in contradiction to palaeontological interpretations (Heissig 1972, 2005, personal communication). Alternatively, there might also be an influence of body mass on the turnover rate of the blood carbonate pool, buffering its isotopic composition from which skeletal apatite forms, as large taxa seem to have lower $\Delta \delta^{13} \mathrm{C}$ and $\Delta \delta^{18} \mathrm{O}$ values (Fig. 3). A further investigation of dental meso- and microwear might allow better constraints on the dietary niches and feeding behavior of the sympatric rhinoceros.

Diet of the suid $H$. soemmeringi

Hyotherium, a relatively small suid, is the most abundant of two suoids in Sandelzhausen and the most common Early 
and early Middle Miocene suoid of Europe (van der Made 2009 , this issue). It is interpreted to have lived in humid and closed environments as it is well known from Miocene brown-coal deposits and associated with swampy woodland settings (van der Made 2009, this issue).

Hyotherium has the highest mean enamel $\delta^{13} \mathrm{C}$ value $(-10.2 \%)$ of all taxa from Sandelzhausen (Fig. 2). This value is about $1.2 \%$ higher than the locality mean $\delta^{13} \mathrm{C}$ value of $-11.4 \%$. Due to differences in digestive physiology the carbon isotope fractionation in suids is slightly smaller than for other large mammals (Harris and Cerling 2002; Passey et al. 2005). Passey et al. (2005) recently determined a ${ }^{13} \mathrm{C}_{\text {diet-enamel }}$ enrichment factor of $13.3 \pm 0.3 \%$ for pigs raised on isotopically controlled diet, which is smaller than the $14.1 \%$ for large ungulate mammals (Cerling and Harris 1999). Using this smaller enrichment factor Hyotherium fed on plants with a $\delta^{13} \mathrm{C}$ value of around $-23.5 \%$, while most other large herbivores fed on plants with $\delta^{13} \mathrm{C}$ values of about $-25 \%$ or lower. This might either indicate feeding in a more open habitat or feeding on canopy-derived fruits or leaves that have higher $\delta^{13} \mathrm{C}$ values than subcanopy plants (Cerling et al. 2004). As Hyotherium is associated with closed, humid environments (van der Made 2009, this issue), feeding in a more open habitat seems unlikely. However, the use of fruits and also scavenging of meat is known from modern African forest hogs (Kingdon 1997; Harris and Cerling 2002). Frugivory of Hyotherium could explain the elevated $\delta^{13} \mathrm{C}$ values; however, frugivory should imply also elevated $\delta^{18} \mathrm{O}$ values as fruits tend to be ${ }^{18} \mathrm{O}$-enriched relative to the source water (Yakir 1997). However, the mean enamel $\delta^{18} \mathrm{O}$ value of Hyotherium is the lowest for all mammal taxa (Fig. 2, Table 2). An omnivorous diet of Hyotherium could explain ${ }^{18} \mathrm{O}$-depleted values relative to the other herbivores because carnivores have lower $\delta^{18} \mathrm{O}$ values than sympatric herbivores (Sponheimer and Lee-Thorp 1999b; Kohn et al. 2005). However, due to a smaller carbon isotope fractionation lower enamel $\delta^{13} \mathrm{C}$ values are to be expected for carnivores, and hence omnivores, compared with sympatric herbivores (Bocherens 2000). Significant consumption of meat by Hyotherium seems unlikely. Therefore a frugivorous diet for Hyotherium is implied, especially as modern frugivorous primates in a rainforest setting have lower enamel $\delta^{18} \mathrm{O}$ values than sympatric folivorous primates (Cerling et al. 2004). Furthermore, the low $\delta^{18} \mathrm{O}$ values indicate a high water dependence of Hyotherium because obligate drinking mammals tend to have the lowest $\delta^{18} \mathrm{O}$ values in terrestrial faunas (Kohn 1996; Levin et al. 2006). This is in good agreement with observations for modern forest hogs being highly water-dependent animals (Harris and Cerling 2002) as well as the palaeontological data indicating a humid, closed environment for Hyotherium (van der Made 2009, this issue).
Diet of the equid A. aurelianense

Anchitherium aurelianense was a brachydont equid with teeth lacking extensive cement. It is found in subtropical to warm-temperate palaeohabitats and is considered as a forest-dwelling browser such as the North American Anchitherium clarencei (MacFadden 2001). However, recent mesowear analyses suggest that Anchitherium at Sandelzhausen was a mixed feeder close to the transition to the browsers, thus being an opportunistic or at least flexible feeder (Kaiser 2009 this issue). Such an opportunistic feeding strategy is supported by the fact that Anchitherium has the highest $\Delta \delta^{13} \mathrm{C}$ value of all taxa from Sandelzhausen (Fig. 3), indicating the use of plant resources with more variable $\delta^{13} \mathrm{C}$ values than the other taxa. The mean $\delta^{13} \mathrm{C}$ value, however, is indistinguishable from those of the largest, probably generalistic, herbivores Lartetotherium and Gomphotherium but also from the cervid Heteroprox (Fig. 2). The mean $\delta^{18} \mathrm{O}$ value and also the $\Delta \delta^{18} \mathrm{O}$ value of Anchitherium are higher than for most other herbivores except for G. fahlbuschi. This supports incorporation of water from more ${ }^{18} \mathrm{O}$-enriched and more varied water sources, which is in accordance with a significant consumption of leaves. Given the tooth morphology, the mesowear signal (Kaiser 2009, this issue) and the enamel carbon and oxygen isotope composition, A. aurelianense from Sandelzhausen probably was a forest or woodland browser, though an intake of some $\mathrm{C}_{3}$ grass cannot be excluded. In conclusion Anchitherium was likely a more flexible feeder than assumed so far.

Diet of the cervid H. eggeri

In the humid Molasse Basin environment usually wet-preferring tragulids are more abundant than cervids; however, Sandelzhausen is an exception with only $10 \%$ of the ruminants being Tragulidae and 90\% Cervidae (Rössner 2004, 2009, this issue). Extensive supply of leaves due to an abundant forest can be assumed because of the high number of five sympatric ruminants at Sandelzhausen (Rössner 2004, 2009, this issue). Based on mesowear data H. eggeri, as well as the sympatric ruminant ungulates Lagomeryx, Dorcatherium, and Germanomeryx were classified as pure browsers (Kaiser and Rössner 2007). The Heteroprox mean enamel $\delta^{13} \mathrm{C}$ value of $-11 \%$ is indistinguishable from those of Lartetotherium, Gomphotherium, and Anchitherium. Thus Heteropox ingested $\mathrm{C}_{3}$ plants with an average $\delta^{13} \mathrm{C}$ value and did not feed in a closed forest environment. Heteroprox has a slightly lower $\delta^{18} \mathrm{O}$ value than most of the other mammals, except for Hyotherium. Therefore, Heteroprox was probably not a canopy browser such as Germanomeryx because if so, higher $\delta^{18} \mathrm{O}$ values would be expected. More likely, Heteroprox was a water dependent 
understory browser in a partially closed forest environment not ingesting large proportions of water from ${ }^{18} \mathrm{O}$-enriched plant tissues such as leaves. However, a high $\Delta \delta^{18} \mathrm{O}$ value indicates that Heteroprox has ingested water with variable $\delta^{18} \mathrm{O}$ values (Fig. 3).

\section{Diet of the palaeomerycid G. fahlbuschi}

The extinct palaeomerycids were ruminants that are comparable to the extant okapi living in dense African rainforests. Palaeomerycidae occur in relative high abundance in Sandelzhausen compared with other Miocene sites in S Germany (Rössner 2004, 2009, this issue). Mesowear data of the extraordinary large palaeomerycid G. fahlbuschi are comparable to the Sumatran rhinoceros and identify the brachydont $G$. fahlbuschi as a browser feeding on soft plants (Kaiser and Rössner 2007; Rössner 2009, this issue) while its metapodial bones are interpreted as an adaptation toward swampy ground (Köhler 1993).

The palaeomerycid G. fahlbuschi has a mean enamel $\delta^{13} \mathrm{C}$ value $(-12 \%)$ lower than the locality mean and than most other mammals. Only the rhinoceros Prosantorhinus and the Metaschizotherium have slightly lower $\delta^{13} \mathrm{C}$ values (Fig. 2). Therefore Germanomeryx was probably feeding in a closed forest which is in good agreement with ecomorphological data (Rössner 2009, this issue). Germanomeryx has the highest mean enamel $\delta^{18} \mathrm{O}$ value $(-5.7 \%$ ) and, together with the cervid Heteroprox, also has the largest range of $\delta^{18} \mathrm{O}$ values (3.2\%o) of all herbivores (Fig. 3). Thus Germanomeryx ingested water from an ${ }^{18} \mathrm{O}$-enriched water source, most likely ${ }^{18} \mathrm{O}$-enriched leaves. Modern giraffes as well as giraffids in Miocene settings generally had higher values relative to other faunal elements (Cerling et al. 1997a) because of their feeding in the upper canopy where leaf water $\delta^{18} \mathrm{O}$ values are high. Therefore, a similar canopy feeding on ${ }^{18} \mathrm{O}$-enriched leaves seems likely for the palaeomerycid G. fahlbuschi.

\section{Diet of the proboscidean G. subtapiroideum}

The proboscideans of Sandelzhausen are dominated by G. subtapiroideum, which makes up $>95 \%$ of the proboscidean remains. Deinotheres are lacking, probably due to ecological reasons as they occur contemporaneously in other sites in the Molasse Basin (Schmidt-Kittler 1972; Göhlich 2009, this issue). For Gomphotherium, mostly juvenile specimens and deciduous teeth are present (Schmidt-Kittler 1972). One deciduous D4 tooth (BSPG II 11326, sample FZ MA SA 2,) that formed preweaning and likely influenced by the consumption of milk, has slightly lower $\delta^{13} \mathrm{C}$ and $\delta^{18} \mathrm{O}$ values than the molars. Milk lipids are fivefold depleted in ${ }^{13} \mathrm{C}$ compared with carbohydrates and proteins (DeNiro and Epstein 1978), thus enamel apatite synthesized before weaning may have lower $\delta^{13} \mathrm{C}$ values than bioapatite synthesized after weaning. The magnitude of this effect depends on the lipid content of the consumed milk. Therefore, this deciduous tooth is excluded from Fig. 2 and the further discussion. Interestingly, the mean $\delta^{13} \mathrm{C}$ value of the tusk enamel samples is about $1 \%$ lower than the mean $\delta^{13} \mathrm{C}$ value of the five molar teeth (Fig. 2). Similar systematic differences between Gomphotherium molar and tusk enamel have been observed for North American Gomphotherium (Fox and Fisher 2004). The reasons for this offset are not clear. Continuously growing tusks and finite-growing molars represent different periods of the ontogeny and may therefore record different dietary compositions. This, however, does not explain why tusk enamel always has lower $\delta^{13} \mathrm{C}$ values compared with molar enamel. An effect from milk consumption on the isotope composition is unlikely as the tip of the tusk is the oldest portion and the basal region which was analyzed (Fig. 4) also formed after weaning. However, the preservation of such small but significant differences in enamel $\delta^{13} \mathrm{C}$ values between tusk and molar enamel indicates that such differences in isotope composition have not been biased by diagenetic alteration. Based on the enamel $\delta^{13} \mathrm{C}$ values G. subtapiroideum was probably a browser or mixed feeder with preference for $\mathrm{C}_{3}$ browse with no seasonal variation in diet, similar as North American Gomphotherium (Fox and Fisher 2004). This is in agreement with its subtapiroidbunodont and brachydont morphology of the cheek teeth and the interpretation relating G. subtapiroideum to a humid woodland biotope (Schmidt-Kittler 1972; Göhlich 2009, this issue).

From one Gomphotherium tusk part of the enamel band was serially sampled (Fig. 4). The sampled interval of $65 \mathrm{~mm}$ of the G. subtapiroideum tusk enamel band probably represents about 1.5 years of tusk growth, assuming a similar tusk growth rate of $45 \mathrm{~mm} /$ year as for Miocene North American Gomphotherium (Fox 2000). The small intratusk variability of the enamel $\delta^{13} \mathrm{C}$ values $(-11.9 \pm 0.1 \%$, $n=16$ ) of the Gomphotherium tusk suggests a fairly constant carbon isotope composition of the $\mathrm{C}_{3}$ plants ingested by this individual over the period of enamel mineralization. No seasonal change in dietary resource use and/or habitat is recorded in the $\delta^{13} \mathrm{C}$ values. Similarly low intratusk $\delta^{13} \mathrm{C}$ variability has been found for North American Gomphotherium (Fox and Fisher 2001, 2004). This might relate to a generalistic, unselective feeding strategy of such a large herbivore or mobility over larger landscape scales integrating a variety of food resources as proboscideans are known to migrate over several $100 \mathrm{~km}$ even on a seasonal basis (Hoppe et al. 1999).

Intratusk $\delta^{18} \mathrm{O}_{\mathrm{CO}_{3}}$ values have only a small range of $1.8 \%$ and display no clear seasonal pattern (Fig. 4). Even if some dampening of the environmental $\delta^{18} \mathrm{O}$ input signal is 
likely due to enamel maturation (Passey and Cerling 2002), no pronounced seasonality is recorded. The proximity of groundwater-fed sources and the large body size may explain a certain isotopic buffering of Gomphotherium body water. Nevertheless, surface drinking water resources used by this Gomphotherium did not have a pronounced seasonal $\delta^{18} \mathrm{O}$ cyclicity. This is in agreement with the warm, subtropical to temperate Middle Miocene climate in S Germany (Böhme 2003, 2009, this study) and the relatively humid conditions in the floodplain environment of Sandelzhausen. In contrast, in the lower part of the Sandelzhausen section a higher seasonality in rainfall occurred (Böhme 2009, this issue) and the formation of pedogenic carbonates indicates a fluctuating groundwater table and seasonally dry periods (Schmid 2002).

\section{Mobility of the large mammals}

The strontium isotope composition $\left({ }^{87} \mathrm{Sr} /{ }^{86} \mathrm{Sr}\right)$ of bones and teeth reflects that of the strontium ingested by the animal with the diet and reflects the ${ }^{87} \mathrm{Sr} /{ }^{86} \mathrm{Sr}$ ratio of the underlying bedrock (Blum et al. 2000; Bentley 2006). The Sr isotope composition is not changed measurably during metabolic processes along the food chain and therefore allows to detect the provenance as well as the migration of animals between isotopically distinct geological domains (Vogel et al. 1990; Hoppe et al. 1999).

The strontium isotope compositions $\quad\left({ }^{87} \mathrm{Sr} /{ }^{86} \mathrm{Sr}=\right.$ $0.710382 \pm 0.0002, n=4$ ) of the Anchitherium tooth and three Gomphotherium teeth is relatively similar and significantly more radiogenic than enamel of other Miocene large mammal teeth from the Molasse Basin realm, which are all below 0.709 (Fig. 5, Tütken et al. 2006). Thus the mammals from Sandelzhausen clearly have ingested their food on isotopically different bed rocks than mammals in the western Molasse Basin. At least the four investigated mammals did not take up large amounts of food in areas with bioavailable ${ }^{87} \mathrm{Sr} /{ }^{86} \mathrm{Sr}$ ratios lower than 0.710 , such as the marine Jurassic limestone rocks of the Swabian and Franconian Alb $\left({ }^{87} \mathrm{Sr} /{ }^{86} \mathrm{Sr} \approx 0.707-0.708\right)$ north of the Molasse Basin, the western Molasse Basin ${ }^{87} \mathrm{Sr} /{ }^{86} \mathrm{Sr} \approx$ 0.708-0.709), or volcanic areas such as the Hegau Province $\left({ }^{87} \mathrm{Sr} /{ }^{86} \mathrm{Sr}<0.704-0.707\right)$ NW of Lake Constanz (Fig. 5). The bioavailable $\mathrm{Sr}$ ingested by these mammals during the period of enamel mineralization thus originates from soils and rocks with high ${ }^{87} \mathrm{Sr} /{ }^{86} \mathrm{Sr}$ ratios $\geq 0.710$. The clastic sediments of the floodplain in the eastern part of the Molasse Basin mostly consist of alpine detritus. Either these sediments have higher bioavailable ${ }^{87} \mathrm{Sr} /{ }^{86} \mathrm{Sr}$ ratios than those in other localities in southern Germany and Switzerland or the crystalline bedrocks of the Bohemian Massive, which outcrop about $50 \mathrm{~km} \mathrm{NE}$ of Sandelzhausen (Fig. 5), were a source of $\mathrm{Sr}$ with high ${ }^{87} \mathrm{Sr} /{ }^{86} \mathrm{Sr}$ ratios for the Sandelzhausen mammals. Therefore, the mammals probably lived most of the time in the realm of the eastern Molasse Basin. However, it is not possible to infer individual home ranges or exact migrational history from the limited data.

\section{Conclusions}

All large herbivorous mammals of the late Early/early Middle Miocene Sandelzhausen fossil site were browsers that fed on $\mathrm{C}_{3}$ plants with an average $\delta^{13} \mathrm{C}$ value of $-25.5 \pm 1 \%$. However, niche partitioning and different habitat use within the humid, low-relief floodplain $\mathrm{C}_{3}$ plant ecosystem is indicated by small but significant intertaxon enamel carbon and oxygen isotope differences. The forest hog H. soemmeringi has the highest $\delta^{13} \mathrm{C}$ and lowest $\delta^{18} \mathrm{O}$ value of all taxa, possibly related to a frugivorous diet. Anchitherium may have had a larger dietary variability than assumed from palaeontological evidence so far. $M$. $b a$ varicum, the small rhinoceros $P$. germanicus, and the palaeomerycid G. fahlbuschi fed in partially closed forests or woodlands. Germanomeryx was probably a canopy browser feeding preferentially on ${ }^{18} \mathrm{O}$-enriched leaves. Most of the mammals were water-dependent browsers in a humid environment as they have similar mean enamel $\delta^{18} \mathrm{O}$ values.

For the late Early/early Middle Miocene (between 16 and $16.5 \mathrm{Ma}$ ) precipitation a $\delta^{18} \mathrm{O}_{\mathrm{H}_{2} \mathrm{O}}$ value of $-5.6 \pm 0.7 \%$ VSMOW can be reconstructed from mammalian enamel $\delta^{18} \mathrm{O}_{\mathrm{PO}_{4}}$ values. The herbivores lived in a warm climate with a MAT of about $19^{\circ} \mathrm{C}$ and low seasonality. Enamel Sr isotope compositions indicate that they most likely ingested their food in the eastern Molasse Basin in the vicinity of the Bohemian Massive and not in the western Molasse Basin or on the Franconian Alb plateau.

Acknowledgments We thank Kurt Heissig, Bayerische Staatssammlung für Paläontologie in Munich for kindly supplying the teeth from Sandelzhausen for isotope sampling. This study was financed by the Swiss National Science foundation grant 200021-100530/1 to TWV and partly by the Emmy Noether-Program of the German National Science Foundation DFG grant TU 148/2-1 to TT. The two reviewers Robert Feranec and Hervé Bocherens provided instructive reviews.

\section{References}

Abdul-Aziz, H., M. Böhme, A. Rocholl, A. Zwing, J. Prieto, J. Wijbrans, R.K. Heissig, and V. Bachtadse. 2008. Integrated stratigraphy and ${ }^{40} \mathrm{Ar} /{ }^{39} \mathrm{Ar}$ chronology of the Early to Middle Miocene Upper Freshwater Molasse in eastern Bavaria (Germany). International Journal of Earth Sciences 97:115-134.

Ayliffe, L.K., and A.R. Chivas. 1990. Oxygen isotope composition of the bone phosphate of Australian kangaroos: Potential as a 
paleoenvironmental recorder. Geochimica et Cosmochimica Acta 54:2603-2609.

Ayliffe, L.K., A.M. Lister, and A.R. Chivas. 1992. The preservation of glacial-interglacial climatic signatures in the oxygen isotopes of elephant skeletal phosphate. Palaeogeography, Palaeoclimatology, Palaeoecology 99:179-191.

Bentley, R.A. 2006. Strontium isotopes from the earth to the archaeological skeleton: A review. Journal of Archaeological Method and Theory 13:135-187.

Blondel, C., H. Bocherens, and A. Mariotti. 1997. Stable carbon and oxygen isotope ratios in ungulate teeth from French Eocene and Oligocene localities. Bulletin de la Société Géologique de France 168:775-781.

Blum, J.D., E.H. Taliaferro, M.T. Weisse, and R.T. Holmes. 2000. Changes in $\mathrm{Sr} / \mathrm{Ca}, \mathrm{Ba} / \mathrm{Ca}$ and ${ }^{87} \mathrm{Sr} /{ }^{86} \mathrm{Sr}$ ratios between two forest ecosystems in the northeastern U.S.A. Biogeochemistry 49:87-101.

Bocherens, H. 2000. Preservation of isotopic signals $\left({ }^{13} \mathrm{C},{ }^{15} \mathrm{~N}\right)$ in Pleistocene mammals. In Biogeochemical approaches to paleodietary analyses, eds. M.A. Katzenberg, and S.H. Ambrose, 65-88. New York: Kluwer/Plenum.

Bocherens, H. 2003. Isotopic biogeochemistry and the paleoecology of the mammoth steppe fauna. In Advances in mammoth research (Proceedings of the second international mammoth conference, Rotterdam, 16-20 May 1999), eds. J.W.F. Reumer, J. De Vos, and D. Mol. Deinsea 9:57-76.

Bocherens, H., P.L. Koch, A. Mariotti, D. Geraads, and J.J. Jaeger. 1996. Isotopic biogeochemistry $\left({ }^{13} \mathrm{C},{ }^{18} \mathrm{O}\right)$ of mammalian enamel from African Pleistocene hominid sites. Palaios 11:306-318.

Böhme, M. 2003. The Miocene climatic optimum: Evidence from ectothermic vertebrates of Central Europe. Palaeogeography, Palaeoclimatology, Palaeoecology 195:389-401.

Böhme, M. 2009. Ectothermic vertebrates (Actinopterygii, Allocaudata, Urodela, Anura, Crocodylia, Squamata) from the Miocene of Sandelzhausen (Germany, Bavaria) and their implication for environment reconstruction and palaeoclimate. Paläontologische Zeitschrift (in press).

Böhme, M., A. Ilg, A. Ossig, and H. Küchenhoff. 2006. New method to estimate palaeoprecipitation using fossil amphibians and reptiles and the middle and late Miocene precipitation gradients in Europe. Geology 34:425-428.

Böhme, M., A. Bruch, and A. Selmeier. 2007. The reconstruction of the Early and Middle Miocene climate and vegetation in the North Alpine Foreland Basin as determined from the fossil wood flora. Palaeogeography, Palaeoclimatology, Palaeoecology 253:91-114.

Bryant, J.D., and P.N. Froelich. 1995. A model of oxygen isotope fractionation in body water of large mammals. Geochimica et Cosmochimica Acta 60:4523-4537.

Bryant, J.D., B. Luz, and P.N. Froelich. 1994. Oxygen isotopic composition of fossil horse tooth phosphate as a record of continental paleoclimate. Palaeogeography, Palaeoclimatology, Palaeoecology 107:303-316.

Bryant, J.D., P.L. Koch, P.N. Froelich, W. Showers, and B.J. Genna. 1996. Oxygen isotope partitioning between phosphate and carbonate in mammalian apatite. Geochimica et Cosmochimica Acta 60:5145-5148.

Cerling, T.E., and J.M. Harris. 1999. Carbon isotope fractionation between diet and bioapatite in ungulate mammals and implications for ecological and paleoecological studies. Oecologia 120:347-363.

Cerling, T.E., Y. Wang, and J. Quade. 1993. Expansion of C4 ecosystems as an indicator of global ecological change in the Late Miocene. Nature 361:344-345.

Cerling, T.E., J.M. Harris, S.H. Ambrose, M.G. Leakey, and N. Solounias. 1997a. Dietary and environmental reconstruction with stable isotope analyses of herbivore tooth enamel from the Miocene locality of Fort Ternan, Kenya. Journal of Human Evolution 33:635-650.

Cerling, T.E., J.M. Harris, B.J. MacFadden, M.G. Leakey, J. Quade, V. Eisenmann, and J.R. Ehleringer. 1997b. Global vegetation change through the Miocene-Pliocene boundary. Nature 389:153-158.

Cerling, T.E., J.M. Harris, and B.H. Passey. 2003a. Diets of East African Bovidae based on stable isotope analysis. Journal of Mammalogy 84:456-470.

Cerling, T.E., J.M. Harris, and M.G. Leakey. 2003b. Isotope paleoecology of the Nawata and Nachukui Formations at Lothagam, Turkana Basin, Kenya. In Lothagam. The Dawn of Humanity in Eastern Africa, eds. J.M. Harris, and M.G. Leakey, 587-597. New York: Columbia University Press.

Cerling, T.E., J.A. Hart, and T.B. Hart. 2004. Stable isotope ecology in the Ituri Forest. Oecologia 138:5-12.

Clementz, M.T., and P.L. Koch. 2001. Differentiating aquatic mammal habitat and foraging ecology with stable isotopes in tooth enamel. Oecologia 129:461-472.

Coombs, M.C. 2009. The chalicothere Metaschizotherium bavaricum (Perissodactyla, Chalicotheriidae, Schizotheriinae) from the Miocene (MN5) Lagerstätte of Sandelzhausen (Germany): Description, comparison, and paleoecological significance. In Fossil lagerstätte Sandelzhausen (Miocene, southern Germany): Contributions to the fauna, eds. G.E. Rössner, and U.B. Göhlich. Paläontologische Zeitschrift 83(1).

Coplen, T.B. 1994. Reporting of stable hydrogen, carbon, and oxygen isotopic abundances. Pure and Applied Chemistry 66:273-276.

Crepet, W.L., and G.D. Feldman. 1991. The earliest remains of grasses in the fossil record. American Journal of Botany 78:1010-1014.

Dansgaard, W. 1964. Stable isotopes in precipitation. Tellus 16:436468.

Deines, P. 1980. The isotopic composition of reduced organic carbon. In Handbook of environmental geochemistry, vol. 1, eds. P. Fritz, and Ch. Fontes, 239-406. New York: Elsevier.

DeNiro, M.J., and S. Epstein. 1978. Influence of diet on the distribution of carbon isotopes in animals. Geochimica et Cosmochimica Acta 42:495-506.

Dettmann, D.L., M. Kohn, J. Quade, F.J. Reyerson, T.P. Ojah, and S. Hamidullah. 2001. Seasonal stable isotope evidence for a strong Asian monsoon throughout the past 10.7 Ma. Geology 29:31-34.

Dongmann, G., H.W. Nürnberg, H. Förstel, and K. Wagener. 1974. On the enrichment of $\mathrm{H}_{2}^{18} \mathrm{O}$ in the leaves of transpiring plants. Radiation and Environmental Biophysics 11:41-52.

Driessens, F.C.M., and R.M.H. Verbeeck. 1990. Biominerals, 440. Boca Raton: CRC.

Drucker, D., H. Bocherens, A. Bridault, and D. Billiou. 2003. Carbon and nitrogen isotopic composition of Red Deer (Cervus elaphus) collagen as a tool for tracking palaeoenvironmental change during Lateglacial and Early Holocene in northern Jura (France). Palaeogeography, Palaeoclimatology, Palaeoecology 195:375388.

Drucker, D.G., A. Bridault, K.A. Hobson, E. Szuma, and H. Bocherens. 2008. Can carbon-13 abundances in large herbivores track canopy effect in temperate and boreal ecosystems? Evidence from modern and ancient ungulates. Palaeogeography, Palaeoclimatology, Palaeoecology 266:69-82.

Ehleringer, J.R., and R.K. Monson. 1993. Evolutionary and ecological aspects of photosynthetic pathway variation. Annual Review of Ecology and Systematics 24:411-439.

Ehleringer, J.R., C.B. Field, Z.F. Lin, and C.Y. Kuo. 1986. Leaf carbon isotope and mineral-composition in subtropical plants along an irradiance cline. Oecologia 70:520-526. 
Ehleringer, J.R., Z.F. Lin, C.B. Field, G.C. Sun, and C.Y. Kuo. 1987. Leaf carbon isotope ratios of plants from a subtropical monsoon forest. Oecologia 72:109-114.

Epstein, S., P. Thompson, and C.J. Yapp. 1977. Oxygen and hydrogen isotopic ratios in plant cellulose. Science 198:1209-1215.

Fahlbusch, V. 2003. Die miozäne Fossil-Lagerstätte Sandelzhausen. Die Ausgrabungen 1994-2001. Zitteliana A 43:109-122.

Fahlbusch, V., and R. Liebreich. 1996. Hasenhirsch und Hundebär. Chronik der tertiären Fossilfundstätte Sandelzhausen bei Mainburg, 40. München: Verlag Pfeil.

Fahlbusch, V., H. Gall, and N. Schmidt-Kittler. 1972. Die obermiozäne Fossil-Lagerstätte Sandelzhausen. 2. Sediment und FossilinhaltProbleme der Genese und Ökologie. Neues Jahrbuch für Geologie und Paläontologie, Monatshefte 1972:331-343.

Fahlbusch, V., H. Gall, and N. Schmidt-Kittler. 1974. Die obermiozäne Fossil-Lagerstätte Sandelzhausen. 10. Die Grabungen 1970-1973 Beiträge zur Sedimentologie und Fauna. Mitteilungen der Bayerischen Staatssammlung für Paläontologie und Historische Geologie 14:103-128.

Farquhar, G.D., J.R. Ehleringer, and K.T. Hubrick. 1989. Carbon isotope fractionation and photosynthesis. Annual Reviews of Plant Physiology and Molecular Biology 44:503-537.

Feranec, R.S. 2007. Stable carbon isotope values reveal evidence of resource partitioning among ungulates from modern $\mathrm{C}_{3}$-dominated ecosystems in North America. Palaeogeography, Palaeoclimatology, Palaeoecology 252:575-585.

Feranec, R.S., and B.J. MacFadden. 2006. Isotopic discrimination of resource partitioning among ungulates in $\mathrm{C}_{3}$-dominated communities from the Miocene of Florida and California. Paleobiology 32:190-205.

Fox, D.L. 2000. Growth increments in Gomphotherium tusks and implications for late Miocene climate change in North America. Palaeogeography, Palaeoclimatology, Palaeoecology 156:327348.

Fox, D.L., and D.C. Fisher. 2001. Stable isotope ecology of a Late Miocene population of Gomphotherium productus (Mammalia, proboscidea) from Port of Entry Pit, Oklahoma, USA. Palaios 16:279-293.

Fox, D.L., and D.C. Fisher. 2004. Dietary reconstruction of Gomphotherium (Mammalia, Proboscidea) based on carbon isotope composition of tusk enamel. Palaeogeography, Palaeoclimatology, Palaeoecology 206:311-335.

Fricke, H.C., and J.R. O'Neil. 1996. Inter- and intra-tooth variation in the oxygen isotope composition of mammalian tooth enamel phosphate; implications for paleoclimatological and paleobiological research. Palaeogeography, Palaeoclimatology, Palaeoecology 126:91-99.

Fricke, H.C., W.C. Clyde, and J.R. O'Neil. 1998. Intra-tooth variations in $\delta^{18} \mathrm{O}\left(\mathrm{PO}_{4}\right)$ of mammalian tooth enamel as a record of seasonal variations in continental climate variables. Geochimica et Cosmochimica Acta 62:1839-1850.

Friedli, H., H. Lotscher, H. Oeschger, U. Siegenthaler, and B. Stauver. 1986. Ice core record of the ${ }^{13} \mathrm{C} /{ }^{12} \mathrm{C}$ ratio of atmospheric $\mathrm{CO}_{2}$ in the past two centuries. Nature 324:237-238.

Göhlich, U.B. 2002. The avifauna of the Miocene Fossillagerstätte Sandelzhausen (Upper Freshwater Molasse, Southern Germany). Zitteliana. Abhandlungen der Bayerischen Staatssammlung für Paläontologie und Geologie 22:169-190.

Göhlich, U.B. 2009. The Miocene proboscidean fauna (mammalia) from Sandelzhausen, southern Germany. In Fossil lagerstätte Sandelzhausen (Miocene, southern Germany): Contributions to the fauna, eds. G.E. Rössner, and U.B. Göhlich. Paläontologische Zeitschrift (in press).

Gregor, H.J. 1982. Zur Ökologie der jungtertiären Säugetier-Fundstelle Sandelzhausen. Documenta Naturae 4:19-26.
Harris, J.M., and T.E. Cerling. 2002. Dietary adaptations of extant and Neogene African suids. Journal of Zoology 256:45-54.

Heaton, T.H.E. 1999. Spatial, species, and temporal variations in the ${ }^{13} \mathrm{C} /{ }^{12} \mathrm{C}$ ratios of $\mathrm{C}_{3}$ plants: Implications for paleodiet studies. Journal of Archaeological Science 26:637-649.

Heissig, K. 1972. Die obermiozäne Fossil-Lagerstätte Sandelzhausen. 5. Rhinocerotidae (Mammalia), Systematik und Ökologie. Mitteilungen der Bayerischen Staatssammlung für Paläontologie und historische Geologie 12:57-81.

Heissig, K. 1997. Mammal faunas intermediate between the reference faunas of MN 4 and MN 6 from the Upper Freshwater Molasse of Bavaria. In Actes du Congrès BiochroM'97, vol. 21, eds. J.-P. Aguilar, S. Legendre, and J. Michaux, 537-546. Mémoires et Travaux de l'Ecole Pratique des Hautes Études, Institut de Montpellier.

Heissig, K. 1999. Family Chalicotheriidae. In The Miocene Land Mammals of Europe, eds. G.E. Rössner, and K. Heissig, 189292. München: Verlag Pfeil.

Hoppe, K.A., P.L. Koch, R.W. Carlson, and S.D. Webb. 1999. Tracking mammoths and mastodons: Reconstruction of migratory behavior using strontium isotope ratios. Geology 27:439442.

Huertas, A.D., P. Iacumin, B. Stenni, B.S. Chillon, and A. Longinelli. 1995. Oxygen isotope variations of phosphate in mammalian bone and tooth enamel. Geochimica et Cosmochimica Acta 59:4299-4305.

Iacumin, P., H. Bocherens, A. Mariotti, and A. Longinelli. 1996. Oxygen isotope analyses of co-existing carbonate and phosphate in biogenic apatite: A way to monitor diagenetic alteration of bone phosphate. Earth and Planetary Science Letters 142:1-6.

Jacobs, B.F., J.D. Kingston, and L.L. Jacobs. 1999. The origin of grassdominated ecosystems. Annals of the Missouri Botanical Garden 86:590-643.

Jechorek, H., and J. Kovar-Eder. 2004. Vegetational characteristics in Europe around the Late Early to Early Middle Miocene based on the plant macro record. In The Middle Miocene Environments and Ecosystem Dynamics of the Eurasian Neogene (EEDEN), vol. 249, eds. F.F. Steininger, J. Kovar-Eder, and M. Fortelius, 53-62. Courier Forschungsinstitut Senckenberg.

Kaiser, T. 2009. Anchitherium aurelianense (Equidae, Mammalia)—a brachydont "dirty browser" in the community of herbivorous large mammals from Sandelzhausen (lowest Middle Miocene, Germany). In Fossil lagerstätte Sandelzhausen (Miocene, southern Germany): Contributions to the fauna, eds. G.E. Rössner, and U.B. Göhlich. Paläontologische Zeitschrift 83(1).

Kaiser, T.M., and G.E. Rössner. 2007. Dietary resource partitioning in ruminant communities of Miocene wetland and karst palaeoenvironments in southern Germany. Palaeogeography, Palaeoclimatology, Palaeoecology 252:424-439.

Kingdon, J. 1997. The Kingdon field guide to African mammals, 450. London: Academic.

Koch, P.L. 2007. Isotopic study of the biology of modern and fossil vertebrates. In Stable isotopes in ecology and envrionmental science, 2nd edn, eds. R. Michener, and K. Lajtha, 99-154. Oxford: Blackwell.

Koch, P.L., D.C. Fisher, and D.L. Dettman. 1989. Oxygen isotopic variation in the tusks of extinct proboscideans; a measure of season of death and seasonality. Geology 17:515-519.

Koch, P.L., N. Tuross, and M.L. Fogel. 1997. The effects of sample treatment and diagenesis on the isotopic integrity of carbonate in biogenic hydroxylapatite. Journal of Archaeological Science 24:417-429.

Köhler, M. 1993. Skeleton and Habitat of recent and fossil Ruminants. Münchner Geowissenschaftliche Abhandlungen A $25: 1-88$ 
Kohn, M.J. 1996. Predicting animal $\delta^{18}$ O: Accounting for diet and physiological adaptation. Geochimica et Cosmochimica Acta 60:4811-4829.

Kohn, M.J., and T.E. Cerling. 2002. Stable isotope compositions of biological apatite. In Phosphates. Geochemical, geobiological, and materials importance, eds. M.J. Kohn, J. Rakovan, and J.M. Hughes. Reviews in Mineralogy and Geochemistry 48:455-488.

Kohn, M.J. 2004. Comment: Tooth enamel mineralization in ungulates: Implications for recovering a primary isotopic time-series, by B.H. Passey and T.E. Cerling 2002. Geochimica et Cosmochimica Acta 68:403-405.

Kohn, M.J., M.J. Schoeninger, and J.W. Valley. 1996. Herbivore tooth oxygen isotope compositions: Effects of diet and physiology. Geochimica et Cosmochimica Acta 60:3889-3896.

Kohn, M.J., M.J. Schoeninger, and J.W. Valley. 1998. Variability in herbivore tooth oxygen isotope compositions: Reflections of seasonality or developmental physiology? Chemical Geology 152:97-112.

Kohn, M.J., M.J. Schoeninger, and W.B. Barker. 1999. Altered states: Effects of diagenesis on fossil tooth chemistry. Geochimica et Cosmochimica Acta 63:2737-2747.

Kohn, M.J., M.P. McKay, and J.L. Knight. 2005. Dining in the Pleistocene-Who's on the menu? Geology 33:649-652.

Lee-Thorp, J.A., and M. Sponheimer. 2005. Opportunities and constraints for reconstructing palaeoenvironments from stable light isotope ratios in fossils. Geological Quarterly 49:195-204.

Lee-Thorp, J.A., and N.J. van der Merwe. 1987. Carbon isotope analysis of fossil bone apatite. South African Journal of Science 83:712-715.

Levin, N.E., T.E. Cerling, B.H. Passey, J.M. Harris, and J.R. Ehleringer. 2006. A stable isotope aridity index for terrestrial environments. Proceedings of the National Academy of Sciences 103:11201-11205.

Longinelli, A. 1984. Oxygen isotopes in mammal bone phosphate: A new tool for palaeoclimatological and palaeoenvironmental research? Geochimica et Cosmochimica Acta 48:385-390.

MacFadden, B.J. 2001. Three-toed browsing horse Anchitherium clarencei from the early Miocene (Hemingfordian) Thomas Farm, Florida. Bulletin of the Florida Museum of Natural History 43:79-109.

MacFadden, B.J., and P. Higgins. 2004. Ancient ecology of 15million-year-old browsing mammals within $\mathrm{C} 3$ plant communities from Panama. Oecologia 140:169-182.

MacFadden, B.J., N. Solounias, and T.E. Cerling. 1999. Ancient diets, ecology, and extinction of 5-million-year-old horses from Florida. Science 283:824-827.

Moser, M., H.-J. Niederhöfer, and G. Falkner. 2009a. Continental molluscs of the fossil site Sandelzhausen (Middle Miocene; Upper Freshwater Molasse from Bavaria) and their value for palaeoecological assessment. In Fossil lagerstätte Sandelzhausen (Miocene, southern Germany): Contributions to the fauna, eds. G.E. Rössner, and U.B. Göhlich. Paläontologische Zeitschrift 83(1).

Moser, M., G.E. Rössner, U.B. Göhlich, M. Böhme, and V. Fahlbusch. 2009b. The fossil lagerstätte Sandelzhausen (Miocene; southern Germany): history of investigation, geology, fauna and age. InFossil lagerstätte Sandelzhausen (Miocene, southern Germany): Contributions to the fauna, eds. G.E. Rössner, and U.B. Göhlich. Paläontologische Zeitschrift 83(1).

Nelson, S.V. 2005. Paleoseasonality inferred from equid teeth and intratooth isotopic variability. Palaeogeography, Palaeoclimatology, Palaeoecology 222:122-144.

Nelson, S.V. 2007. Isotopic reconstructions of habitat change surrounding the extinction of Silvapithecus, a Miocene hominoid, in the Siwalik Group of Pakistan. Palaeogeography, Palaeoclimatology, Palaeoecology 243:204-222.
O'Leary, M.H. 1988. Carbon isotopes in photosynthesis. Bioscience 38:328-336.

Passey, B., and T.E. Cerling. 2002. Tooth enamel mineralization in ungulates: Implications for recovering a primary isotopic timeseries. Geochimica et Cosmochimica Acta 66:3225-3234.

Passey, B.H., T.E. Cerling, M.E. Perkins, M.R. Voorhies, J.M. Harris, and S.T. Tucker. 2002. Environmental change in the great plains: An isotopic record from fossil horses. The Journal of Geology 110:123-140.

Passey, B.J., T.F. Robinson, L.K. Ayliffe, T.E. Cerling, M. Sponheimer, M.D. Dearing, B.L. Roeder, and J.R. Ehleringer. 2005. Carbon isotope fractionation between diet, breath $\mathrm{CO}_{2}$, and bioapatite in different mammals. Journal of Archaeological Science 32:1459-1470.

Peter, K. 2002. Odontologie der Nashornverwandten (Rhinocerotidae) aus dem Miozän (MN 5) von Sandelzhausen (Bayern). Zitteliana. Abhandlungen der Bayerischen Staatssammlung für Paläontologie und Geologie 22:3-168.

Quade, J., T.E. Cerling, J.C. Barry, M.E. Morgan, D.R. Pilbeam, A.R. Chivas, J.A. Lee-Thorp, and N.J. van der Merwe. 1992. A 16-Ma record of paleodiet using carbon and oxygen isotopes in fossil teeth from Pakistan. Chemical Geology 94:183-192.

Quade, J., T.E. Cerling, P. Andrews, and B. Alpagut. 1995. Paleodietary reconstruction of Miocene faunas from Pasalar, Turkey using stable carbon and oxygen isotopes of fossil tooth enamel. Journal of Human Evolution 28:373-384.

Rössner, G.E. 2004. Community structure and regional patterns in late Early to Middle Miocene Ruminantia of Central Europe. In The Middle Miocene environments and ecosystem dynamics of the Eurasian neogene (EEDEN), vol. 249, eds. F.F. Steininger, J. Kovar-Eder, and M. Fortelius, 91-100. Courier ForschungsInstitut Senckenberg.

Rössner, G.E. 2009. Systematics and palaeoecology of the ruminant (Artiodactyla, Mammalia) community from Sandelzhausen (Early/Middle Miocene boundary) in the German Molasse Basin. In Fossil lagerstätte Sandelzhausen (Miocene, southern Germany): Contributions to the fauna, eds. G.E. Rössner, and U.B. Göhlich. Paläontologische Zeitschrift (in press).

Rozanski, K., L. Araguás-Araguás, and R. Gonfiantini. 1993. Isotopic patterns in modern global precipitaion. In Climate Change in continental isotopic records, eds. P.K. Swart, K.C. Lohmann, J. McKenzie, and S. Savin. Geophysical Monograph 78:1-36.

Schmid, W. 2002. Ablagerungsmilieu, Verwitterung und Paläoböden feinklastischer Sedimente der Oberen Süßwassermolasse Bayerns. Abhandlungen der Bayerischen Akademie der Wissenschaften, Mathematisch-Naturwissenschaftliche Klasse, Neue Folge 172:207.

Schmidt-Kittler, N. 1972. Die obermiozäne Fossil-Lagerstätte Sandelzhausen. 6. Proboscidea (Mammalia). Mitteilungen der Bayerischen Staatssammlung für Paläontologie und Historische Geologie 12:83-95.

Schoeninger, M.J., and M.J. DeNiro. 1982. Carbon isotope ratios of apatite from fossil bone cannot be used to reconstruct diets of animals. Nature 297:557-578.

Schulz, E., and J.M. Fahlke. 2009. The diet of Metaschizotherium bavaricum (Chalicotheriidae, Mammalia) from the MN5 of Sandelzhausen (Germany) implied by the mesowear method. In Fossil lagerstätte Sandelzhausen (Miocene, southern Germany): Contributions to the fauna, eds. G.E. Rössner and U.B. Göhlich. Paläontologische Zeitschrift 83(1).

Schulz, E., J.M. Fahlke, G. Merceron, and T. Kaiser. 2007. Feeding ecology of the Chalicotheriidae (Mammalia, Perissodactyla, Ancylopoda). Results from dental micro- and mesowear analyses. Verhandlungen des Naturwissenschaftlichen Vereins Hamburg 43:5-31. 
Schweigert, G. 1992. Die untermiozäne Flora (Karpatium, MN5) des Süßwasserkalkes von Engelswies bei Meßkirch (Baden-Württemberg). Stuttgarter Beiträge zur Naturkunde, Serie B 188:155.

Sharp, Z.D., and T.E. Cerling. 1998. Fossil isotope records of seasonal climate and ecology: Straight from the horse's mouth. Geology 26:219-222.

Sponheimer, M., and J.A. Lee-Thorp. 1999a. Isotopic evidence for the diet of an early hominid, Australopithecus africanus. Science 283:368-370.

Sponheimer, M., and J.A. Lee-Thorp. 1999b. Oxygen isotope in enamel carbonate and their ecological significance. Journal of Archaeological Science 26:723-728.

Spötl, C., and T.W. Vennemann. 2003. Continuous-flow IRMS analysis of carbonate minerals. Rapid Communications in Mass Spectrometry 17:1004-1006.

Sternberg, L.S.L. 1989. Oxygen and hydrogen isotope ratios in plant cellulose: Mechanisms and applications. In Stable isotopes in ecological research, eds. P.W. Rundel, J.R. Ehleringer, and K.A. Nagy, 124-143. New York: Springer.

Tidwell, W.D., and E.M.V. Nambudiri. 1989. Tomlinsonia thomassonii, gen. et sp. nov., a permineralized grass from the upper Miocene Ricardo Formation. California. Reviews of Paleobotany and Palynology 60:165-177.

Tieszen, L.L., and T. Fagre. 1993. Effect of diet quality and composition on the isotopic composition of respiratory $\mathrm{CO}_{2}$, bone collagen, bioapatite, and soft tissues. In Prehistoric human boned archaeology at the molecular level, eds. J.B. Lambert, and G. Grupe, 121-155. Berlin: Springer.

Tütken, T., T.W. Vennemann, H. Janz, and E.P.J. Heizmann. 2006. Palaeoenvironment and palaeoclimate of the Middle Miocene lake in the Steinheim basin, SW Germany, a reconstruction from $\mathrm{C}, \mathrm{O}$, and $\mathrm{Sr}$ isotopes of fossil remains. Palaeogeography, Palaeoclimatology, Palaeoecology 241:457-491.

van der Made, J. 2009. The pigs and "Old World peccaries" (Suidae and Palaeochoeridae, Suoidea, Artiodactyla) from the Middle Miocene of Sandelzhausen (southern Germany)—phylogeny and an updated classification of the Hyotheriinae and Palaeochoeridae. In Fossil lagerstätte Sandelzhausen (Miocene, southern Germany): Contributions to the fauna, eds. G.E. Rössner, and U.B. Göhlich. Paläontologische Zeitschrift (in press).

van der Merwe, N.J., and E. Medina. 1989. Photosynthesis and ${ }^{13} \mathrm{C} /{ }^{12} \mathrm{C}$ ratios in Amazonian rain forests. Geochimica et Cosmochimica Acta 53:1091-1094.

van der Merwe, N.J., and E. Medina. 1991. The canopy effect, carbon isotope ratios and foodwebs in Amazonia. Journal of Archaeological Science 18:249-259.

Vennemann, T.W., H.C. Fricke, R.E. Blake, J.R. O'Neil, and A. Colman. 2002. Oxygen isotope analysis of phosphates: A comparison of techniques for analysis of $\mathrm{Ag}_{3} \mathrm{PO}_{4}$. Chemical Geology 185:321-336.

Vogel, J.C., B. Eglington, and J.M. Auret. 1990. Isotope fingerprints in elephant bone and ivory. Nature 346:747-749.

Wang, Y., and T. Cerling. 1994. A model of fossil tooth and bone diagenesis: Implications for paleodiet reconstruction from stable isotopes. Palaeogeography, Palaeoclimatology, Palaeoecology 107:281-289.

Witt, W. 1998. Die miozäne Fossil-Lagerstätte Sandelzhausen. 14. Ostracoden. Mitteilungen der Bayerischen Staatssammlung für Paläontologie und Historische Geologie 38:135-165.

Yakir, D. 1992. Variations in the natural abundances of oxygen-18 and deuterium in plant carbohydrates. Plant, Cell, and Environment 15:1005-1020.

Yakir, D. 1997. Oxygen-18 of leaf water: A crossroad for plant associated isotopic signals. In Stable isotopes: Integration of biological, ecological. and geochemical processes, eds. $\mathrm{H}$. Griffiths, 147-168. Oxford: BIOS.

Zachos, J., M. Pagani, L. Sloan, E. Thomas, and K. Billups. 2001. Trends, rhythms, and aberrations in global climate $65 \mathrm{Ma}$ to present. Science 292:686-693.

Zanazzi, A., and M. Kohn. 2008. Ecology and physiology of White River mammals based on stable isotope ratios of teeth. Palaeogeography, Palaeoclimatology, Palaeoecology 257:2237. 